\title{
Cardiac cell modelling: Observations from the heart of the cardiac physiome project
}

\author{
Martin Fink $^{\mathrm{a}^{*}}$, Steven A. Niederer ${ }^{\mathrm{b}^{*}}$, Elizabeth M. Cherry ${ }^{\mathrm{c}}$, Flavio H. Fenton ${ }^{\mathrm{c}}$, Jussi T. \\ Koivumäki $^{\mathrm{d}}$, Gunnar Seemann ${ }^{\mathrm{e}}$, Rüdiger Thul ${ }^{\mathrm{f}}$, Henggui Zhang ${ }^{\mathrm{g}}$, Frank B. Sachse ${ }^{\mathrm{h} * *}$, Dan \\ Beard $^{\mathrm{i} * *}$, Edmund J. Crampin ${ }^{\mathrm{j} * *}$, Nicolas P. Smith ${ }^{\mathrm{b} * 1}$
}

${ }^{a}$ Department of Physiology, Anatomy and Genetics, University of Oxford, OX1 3JP, United Kingdom, now: Modeling and Simulation, Novartis Pharma AG, 4002 Basel, Switzerland

${ }^{\mathrm{b}}$ Computing Laboratory, University of Oxford, OX1 3QD, United Kingdom

${ }^{c}$ Department of Biomedical Sciences, Cornell University, Ithaca, NY 14850, USA

${ }^{\mathrm{d}}$ Department of Physics, P.O. Box 3000, 90014 University of Oulu, Finland

${ }^{\mathrm{e}}$ Institute of Biomedical Engineering, Karlsruhe Institute of Technology, 76131 Karlsruhe, Germany

${ }^{\mathrm{f}}$ School of Mathematical Sciences, University of Nottingham, NG7 2RD, United Kingdom

${ }^{\mathrm{g}}$ Biological Physics Group, School of Physics and Astronomy, The University of Manchester, Manchester M13 9PL, UK

${ }^{\mathrm{h}}$ Nora Eccles Harrison Cardiovascular Research and Training Institute and

Bioengineering Department, University of Utah, Salt Lake City, UT 84112, USA

${ }^{\mathrm{i}}$ Biotechnology and Bioengineering Center, Dept. of Physiology, Medical College of Wisconsin, Milwaukee, WI, USA

${ }^{\mathrm{j}}$ Auckland Bioengineering Institute and Department of Engineering Science, The University of Auckland, Private Bag 92019, Auckland 1142, New Zealand

* Joint first authors

${ }^{* *}$ Joint senior authors

Keywords: Cardiac, Cellular, Physiome, Electrophysiology, Mathematical Modelling

In this manuscript we review the state of cardiac cell modelling in the context of international initiatives such as the IUPS Physiome and Virtual Physiological Human Projects, which aim to integrate computational models across scales and physics. In particular we focus on the relationship between experimental data and model parameterisation across a range of model types and cellular physiological systems. Finally, in the context of parameter identification and model reuse within the Cardiac Physiome, we suggest some future priority areas for this field.

\section{Introduction}

The Physiome Project is a worldwide effort that aims to produce an integrative computational framework with which to understand eukaryotic and human physiology (Crampin et al. 2004 and Hunter and Borg 2003). Formalised under the banners of the International Union of Physiological Sciences (IUPS) (Hunter 2004) and Virtual

\footnotetext{
${ }^{1}$ Corresponding Author: nic.smith@comlab.ox.ac.uk (Nic Smith)
} 
Physiological Human (VPH) (Viceconti et al. 2008) initiatives, the approach and philosophy are common to a large number of computational physiology research efforts.

Multiscale heart modelling is arguably further advanced than any other organ system; in particular the modelling of cardiac contraction, electrical excitation and metabolism at the cellular scale provides perhaps the most sophisticated examples of modelling frameworks currently available. Most notably, within the class of cell and tissue models dealing with cardiac electrophysiology, multiple data sets and functional knowledge have been rationalised and incorporated into single-cell modelling frameworks. These frameworks have in turn provided the ability to analyse the effects of the multiple coupled mechanisms which underpin the emergent behaviour of the heart (Rudy 2000).

The inherently modular structure of cell physiology has been central to the progression of this cellular modelling work and perhaps the Physiome concept itself. Modelling the channels and transporters which regulate ionic flux across cellular membranes as individual components provides a set of discrete sub-models. These sub-models are often phenomenological at the scale of their biochemical regulation (described by kinetic schemes which are fitted to data). However, by coupling sub-models describing regulation of ionic fluxes within and between sub-spaces (e.g. buffers, channels and exchangers), whole cell models can be assembled, capturing the electrophysiological organisation of the cell. This modular structure, both in vivo and in silico, provides the potential to uncouple these physiological components and to facilitate the development and assembly of the sub-models which describe them. Specifically, new cell models can be created through introducing new components into existing cellular modelling frameworks or by assembling existing components into a new cellular biophysical structure (e.g. Terkildsen et al. 2008 and Cortassa et al. 2003). The cardiac cellular modelling literature has many examples of model development through both processes, often in combination, i.e. individual components are replaced (e.g. Flaim et al. 2006) and frameworks are altered (e.g. Campbell et al. 2009). The success of this approach is evident in the expanding number of cardiac models with increasing focus on representing specific tissue types, animal species and physiological functions; many of these are reviewed below.

The relative maturity of cardiac cell modelling has meant that developments in this field have shaped many of the ideas fundamental to the philosophy of the Physiome, in particular, using biophysical models for integrating data from multiple sources, the development of multiscale models through coupling existing model components and using interfaces that represent specific biophysical mechanisms. Through the development of community standards for unambiguously representing and curating models, we now have the ability to rapidly and accurately assemble and couple functional representations of physiological processes. Through this framework, we gain in turn the capacity to embed multiple layers of knowledge to reveal insights and account for emergent or otherwise unanticipated effects. Examples of such insights in the cardiac field include the early identification of the energy-saving properties of the inward potassium rectifier current (Noble 1962) through to more recent electro-mechanical 
models which predicted spatial heterogeneity (Campbell et al. 2008) before it was subsequently confirmed experimentally (Stelzer et al. 2008).

However, the relative maturity of computational modelling of cardiac cellular function means that, in addition to being in a unique position to illustrate the power of the Physiome approach, it also highlights most tangibly some of the most significant current challenges. In our experience, many of these issues also relate to the reuse of model components (Niederer et al. 2009). Specifically, while markup languages such as CellML (Garny et al. 2008) and SBML (Hucka et al. 2004) allow models to be reused with fidelity, there is no clear way for the assumptions and data on which individual model components are based to also be inherited. This means there is potential for existing submodels to be embedded in new frameworks which aim to simulate a dynamic range inappropriate for the initial parameterisation of the model component. It also means that errors that were made when an existing sub-model was developed, for example in the kinetic scheme or parameter fit of a channel or exchanger, are carried through 'as is' when building a new cell model. Furthermore, because in some cell models practitioners have determined parameters by fitting the whole cell model to emergent measures of cell function, such as action potentials or tension transients, errors that this introduces in submodels are propagated when components of these models are reused. In cases where the emergent behaviour is not sensitive to error in a particular sub-component this is not an important issue. However, in at least some cases this will not be true and the sensitivity of a particular physiological property to parameter errors will need to be considered during model development. This is, apparently, rarely done at present.

Many modelling papers do not provide any details about the method used for determining the model parameters; some reveal that the fit was done by hand. This may indicate a lack of software tools to guide modellers in this task. More drastically, physiological conclusions have been drawn from models that apparently do not provide a good fit to the experimental data (e.g., Fink and Noble 2009). Another problem is that the experimental data ultimately underlying the parameterisation of whole cell models do not always comprise a consistent data set, i.e., the data have been collected from different cell types and species, and under various experimental conditions, including substantial variations in temperature. Any model derived from, and able to fit all these data at once would only describe some mean, relatively nonspecific behaviour. It is important to note that data may not always be consistent between, or even within, experimental laboratories and/or preparations. However, species and temperature consistency is, arguably, a fundamental step in enabling models to quantitatively and mechanistically represent physiological function. In fact, different models of cell electrophysiology for the same species and region of the heart tend to show different behaviour in terms of responses to channel block and in emergent properties like rate adaptation and memory (e.g. Nygren et al. 2001, ten Tusscher et al. 2006, Cherry and Fenton 2007, Bueno-Orovio et al. 2008, Cherry et al. 2008).

Finally, the parameter identification problem is itself also a key issue in managing the link between cellular model parameters and experimental data. Specifically, the challenge is in determining and quantifying the ability to uniquely extract a parameter value from 
available experimental data. By reusing model components this process of determining parameters from data can be avoided, although it is unclear to what extent parameter values for different types of cells and across species remain the same. However, within cardiac cell models, inheritance of model components has recently been shown to span multiple generations of models meaning that these components, and thus also the models that contain them, are often based at least in part on data collected many years before the model was published (Niederer et al. 2009). For example, older models routinely relied on multi-cellular preparation data, which, in many cases, have now been superseded by isolated cell data. Furthermore, species and/or temperature-specific data may not have been available for earlier models but is now more readily available. Through incautious model reuse, parameters derived from data which are now superseded can find their way into newly published models. With new experimental producing an increasing quality and quantity of measurement data, this represents an opportunity lost.

Our goals for this manuscript are multiple. For the reader new to the subject, in Section 2 we outline the sources of experimental data used to parameterise equations representing specific cellular processes, described in Section 3. In Section 4 we review different classes of cardiac cell models before, in Section 5, discussing research issues relating to model identification and parameterisation and the need, arising from this, to robustly link model parameters and data in the form of standardised tests. Finally in Section 6 we conclude with some proposals for potential future initiatives for the field.

\section{Experimental Preparations and Techniques}

As outlined above, models of cardiac cells and sub-cellular components are based on numerous experimental studies carried out on various types of preparations. Data from these studies are used for guiding the design of model structure as well as for parameterisation of model components and validation. This section will introduce the major types of experimental preparations and measurement techniques. Attention is paid primarily to preparations and techniques at the cellular, sub-cellular and molecular levels that are currently providing the major data source for cardiac cell modelling.

\subsection{Experimental Preparations}

A major decision in the design of experimental studies is the selection of an appropriate preparation. This selection has important implications for the interpretation of data from the experimental study and also the application of the data in modelling. Across the range of scales from protein-expression systems through to cell-membrane patches and isolated cells to tissue sections, established types of preparations have been developed. Most preparations for cardiac studies are harvested from excised animal hearts. Human preparations are commonly obtained by biopsy and from unused heart transplants.

At the protein level several expression systems have been developed to study the biochemistry and function of proteins. The systems allow for expressing exogenous proteins in cell types such as frog oocytes, human embryonic kidney (HEK) cells and immortalised monkey kidney cells (COS). Protein expression is caused by injection of mRNA into these cells. The mRNA can be modified by site-directed mutagenesis, which allows for characterisation of the effects of mutations. A feature of expression systems is 
the commonly high level of protein expression, which allows one not only to neglect the effects of native proteins (expressed at lower levels), but also to increase the signal-tonoise ratio of measurement data. Thus, expression systems facilitate measurements of signals that are not apparent in native cells and/or cannot be distinguished from other signals. An example is the measurement of gating currents of ion channels (Armstrong and Bezanilla 1973), which have amplitudes smaller than $1 \%$ of the ion current passing through the channels. A limitation of expression systems is that the protein is not expressed in its native environment, which would include auxiliary proteins and microspaces.

Membrane patches can be extracted from many cell types using techniques developed by Neher and Sakmann (2007). These microscopic patches provide the basis for a number of measurement approaches to studying the electrophysiology of ion channels, transporters and exchangers (see next section) and the characterisation of related membrane proteins and signalling pathways. Membrane patches are commonly extracted from isolated cells, which constitute the major preparation for studies of the properties of single cells. An established method to obtain isolated cell preparations is based on dissociating cells from tissue by collagenases (enzymes that degrade collagen). These preparations of isolated cells are applied, for instance for recording electrical currents and voltages through cell membranes. Isolated cells are also a standard preparation for microscopic imaging studies of cell architecture and function, e.g. intracellular calcium concentration and membrane voltage. A problem with isolated-cell preparations is the removal of intercellular coupling and coupling to the extracellular matrix, which are well-established modulators of cell function. To address this issue, recent modifications of cell-isolation protocols have been developed to provide for preparations of isolated myocyte pairs (Zaniboni et al. 2003). This type of preparation provides a valuable resource for studies of intercellular coupling and the involved proteins, in particular connexins.

Various preparations at tissue level have been developed for cardiac studies. Established tissue preparations include trabeculae, papillary muscle, stripes of atrial tissue and ventricular wedges. Depending on preparation size and experimental protocols, maintaining viability of these preparations necessitates specific consideration. While the application of tissue preparations has limitations in their ability to provide information at cellular and sub-cellular level, the preparations can offer a more native environment for the study of various processes at this level. Other preparation types are based on cell culture and tissue engineering. Commonly, a selection of isolated cells from neonatal animals is applied. A specific feature of this preparation is its long-term availability, which allows for monitoring of developmental and remodelling phenomena.

\subsection{Experimental Techniques}

\subsubsection{Protein Structure and Expression}

Structures of proteins at sub-nanometre resolution can be determined with X-ray crystallography and NMR spectroscopy. Structural models relevant to the modelling in this review include those for ion channels, actin and myosin. Homology modelling allows the construction of models of related proteins based on known structures. The underlying assumptions are that structures are conserved and amino acid sequences are similar in 
related proteins. Structural models can provide guidance for biophysically motivated design of models. Structural models are also used in molecular dynamics simulations, for instance of binding of drugs to ion channels. The relationship of these atom-level modelling approaches to sub-cellular and cellular modelling is further discussed in Section 3.1.

Detection of protein expression and qualitative assessment of expression levels are commonly carried out with the western blot method. The method can be applied to all the types of experimental preparation outlined above. The method requires antibodies specific to the protein of interest. Major applications of the method include testing whether proteins are expressed in a specific cell, determining whether protein expression is altered, for instance in disease, and providing for qualitative relationships between expression levels in different cell or tissue types. Limitations of the western blot method are associated with its qualitative character and the specificity of antibodies.

\subsubsection{Sub-cellular and Cell Architecture}

Optical and electron microscopy are the major techniques used to gain insights into subcellular and cell architecture. A major difference between the two techniques is their image resolution. Resolution of electron microscopy is in the sub-nanometre range whereas the resolution of optical microscopy is in the sub-micrometre range (Bolte and Cordelières 2006). However, novel developments indicate that significant improvements can be achieved for optical microscopy (Soeller et al. 2009). While both techniques initially focused on two-dimensional imaging, confocal microscopy and wide-field microscopy together with deconvolution methods and 3D electron microscopy facilitate volumetric characterisation of preparations. The obtained microscopic data provide a structural basis for cellular modelling in a similar manner to how magnetic resonance imaging and computed tomography provide image data for macroscopic modelling of the heart. Commonly, image processing and visualisation methods are necessary to produce models of cells and sub-cellular structure.

Methods such as confocal microscopy commonly take advantage of fluorescent dyes conjugated to antibodies for proteins or other markers. Confocal imaging systems allow the application and imaging of various dyes simultaneously, e.g. to study co-localisation of proteins and enrich functional data with structural information. A general advantage of optical microscopy is that it allows for functional imaging, e.g. of transmembrane voltage, intracellular calcium and $\mathrm{pH}$, and can be applied to in vivo preparations. Recent technological advances in catheter-based confocal microscopy and local dye delivery promise in vivo imaging of cells inside heart tissue in situ at sub-micrometre resolution (Lasher et al. 2009).

\subsubsection{Electrophysiology}

Methodologies for electrophysiological characterisation of cardiac preparations at the cellular and sub-cellular levels comprise patch-clamp techniques on isolated membranes and cells, recordings of membrane voltages of cells in tissue using piercing microelectrodes, and microscopic imaging with functional dyes. Patch-clamp techniques as introduced above are the primary approach for studying membranes and their associated 
proteins (Neher and Sakmann 2007). The techniques are based on micropipettes, which are used for extraction of patches from cell or organelle membranes as well as serving as electrodes for the application and measurement of voltages across the membrane and currents through it. The techniques allow for measurement of currents of single ion channels, demonstrating their stochastic behaviour. Whole-cell recordings can be based on a similar approach to that for patch-clamping. The recordings involve the contribution of various channels, exchangers and transporters over the whole membrane. Both patchclamp techniques and whole-cell recordings also play an important role in the characterisation of cells from protein expression systems.

Standard protocols for characterisation of electrophysiology of membranes and proteins include voltage stepping, e.g. to assess activation, deactivation and inactivation of ion channels. Action potential clamps have been used for characterisation of the contribution of specific currents during an action potential (deHass and Vogel 1989) and for studying intracellular calcium dynamics (Chudin et al. 1999).

Recordings using micro-electrodes piercing through cell membranes provide information about the membrane voltages of a single cell that can remain coupled to other cells in a tissue preparation. One of the main features of this approach is that it is possible to obtain action potentials from cells that are still coupled. Similarly to the preparations discussed above, the tissue preparations can remain viable for hours, which allows a significant amount of data acquisition through a wide range of experimental conditions and protocols. Commonly, properties of sub-cellular components cannot be measured with this technique.

Various fluorescent dyes have been developed for imaging of membrane voltages and calcium concentrations in cells and cell compartments (Fluhler et al. 1985, Nilius 1985, Matiukas et al.2006). Voltage-sensitive dyes reside in the membrane and report changes in the membrane voltage by changes in their emitted light spectra. A major application of calcium dyes is the characterisation of mechanisms underlying excitation-contraction coupling in myocytes (Cheng et al. 1993). Commonly, these functional dyes are used for imaging with confocal microscopy. The imaging approach promises to provide detail on the spatio-temporal heterogeneity of functions such as intracellular calcium transients. Current limitations include reduced temporal resolution in comparison to electrical measurements, issues with the toxicity of dyes and their effects on cell physiology.

\subsection{Current Experimental Challenges and Limitations}

There are various limitations to experimental preparations, techniques and data. These limitations should be accounted for in model development and application. A major limitation is the deterioration of most of the previously described experimental preparations. Deterioration can affect both structure and function of preparations. Deterioration can occur over time scales that can be close to or even smaller than the time scale of experimental data acquisition. Furthermore, isolation of cells and tissues (see above) can accelerate deterioration and cause further damage, thereby affecting normal structure and function. Isolation of cells and tissue also removes various environmental stimuli, such as mechanics and hormones, which are known to modulate 
electrophysiology. Development of reliable experimental preparations remains a challenge, in particular for studies of long-term developmental and disease processes.

In general, data measured with the previously described experimental techniques are affected by various measurement artefacts, for instance noise from various sources intrinsic or extrinsic to the experimental preparation. The frequency response properties of the measurement system, discretisation of temporal and spatial signals, and processing of data such as filtering can introduce further artefacts. These artefacts can complicate data analysis and interpretation. Other limitations of experimental data are related to the separability of signals and extraction of signals from the component of interest. For instance, voltage-step protocols modulate the electrophysiology of various components in a myocyte. Application of blockers and activators, pipette and bath solutions with appropriate ion concentrations and specifically designed experimental protocols can attenuate this problem. However, their application can also affect the electrophysiology of the component of interest.

Application of data in modelling studies necessitates various selection and design decisions. Detailed knowledge of experimental preparations, techniques and measurement conditions is necessary for parameterisation and integration of models. Measurement conditions that are requisite, for example in the development of ion channel models, include temperature, $\mathrm{pH}$ and ion concentrations in the intra- and extracellular solutions.

The focus of most cardiac experimental studies is on the characterisation of basic physiological mechanisms in animals. Experimental preparations of disease and aging in various species have been developed, but the data available from those preparations is much less comprehensive in comparison to the amount and detail of physiological data. The extent of human data of cellular and sub-cellular process, in particular in disease and aging, is even smaller. These deficits mean that even state-of-the-art cellular and subcellular human models are still based on a significant amount of animal data.

\section{Model Component Development}

Ideally, model design and parameters would be derived from well-known and tested biophysical principles from a smaller scale than the model scale - in the case of ionchannel models this would mean deriving model design and parameters from molecular dynamic simulations of the ion-channel protein. As this is currently impossible, it is necessary to develop hybrid models (connecting microscale with mesoscale), i.e. models that are constructed partially from knowledge of the molecular structure (see Section 2.2.1), but fitted to data at the same scale, for example gating currents, single channel measurements or whole cell current data (see Section 2.2.3). For models of contraction and intracellular calcium dynamics not only the protein functions but also their localisation is important. For calcium models in particular it is essential to have knowledge about the localisation of calcium stores and release units from data on subcellular structures in addition to the information on molecular structures and electrophysiological data (see Section 2.2.2). 


\subsection{Models for Ion Channels}

The major approach for quantitative description of ion channels and other proteins is Markov chain-type models. This approach allows description of memory-less processes, i.e. only the present state of a process is known and used for the calculation of subsequent states.

A Markov model consists of a set of states and a further set of equations describing the transitions between these states. The states can be considered as discrete configurations of proteins, for instance, the open and closed state of an ion channel. Transitions between states can be dependent on various quantities including voltage, temperature and drug concentrations. For example, a simple two-state Markov model is defined as:

$$
C \underset{\bar{\beta}}{\stackrel{\alpha}{-}} O
$$

with the states $\mathrm{C}$ and $\mathrm{O}$. The transitions between these states are described by the rate coefficients $\alpha$ and $\beta$. Consideration of a population of such channels allows a continuum limit to be taken in which the time evolution of population state occupancy probabilities, and in particular the open probability $p_{0}$, can be described by ordinary differential equations (ODEs) - leading, for example, to the Hodgkin-Huxley type models originally developed to describe the gating of sodium and potassium channels in the squid giant axon from experimental data.

Knowledge of protein function and structure is commonly applied in Markov-model design to determine the set of states and the regulation and rate of transitions between each state. For instance, the tetrameric structure of Shaker potassium channels, with four independently acting voltage sensors, motivated the design of a 15-state model of gating currents (Section 2). Structural considerations can also limit the number of free parameters, e.g. the number of rate coefficients in the mentioned model of gating currents. Further constraints may arise from thermodynamic considerations leading, for example to imposition of a detailed balance on the kinetic parameters. However, the tension between the complexity of a model framework required to replicate biophysical structure and the ability to define parameters from experimental data is arguably most apparent in the use of Markov models. Specifically, motivated by knowledge of protein structure, Markov models can consist of a large number of states and transitions, which may be excessive from an information theoretical point of view. For example, the transient outward $\mathrm{K}^{+}$current $\mathrm{I}_{\mathrm{to}}$ has been modelled using ten-state Markov models for each of the two components ( $\mathrm{I}_{\mathrm{Kv} 4.3}$ and $\left.\mathrm{I}_{\mathrm{Kv} 1.4}\right)$, leading to a total of 20 states to describe $\mathrm{I}_{\text {to }}$ (Iyer et al. 2004).

A further modelling step is commonly necessary to connect Markov models of protein dynamics with functional or measured data. For example, a model of ion fluxes through the channel pore is necessary to describe the currents measured in voltage clamp experiments. To a first approximation, Ohm's law can be used to calculate the fluxes caused by voltage and concentration gradients (Hodgkin and Huxley 1952):

$$
I_{X}=N p_{o} g_{X}\left(V_{m}-E_{X}\right)
$$


with $\mathrm{I}_{\mathrm{X}}$ the ion current of ion type $\mathrm{X}, \mathrm{N}$ the number of channels in the membrane, $\mathrm{p}_{\mathrm{o}}$ the open probability, $g_{X}$ the maximal conductance of a single channel, $V_{m}$ the transmembrane voltage and $E_{X}$ the Nernst potential of ion type $X$. Depending on channel and ion type, another description of current flow might be more appropriate, i.e. the Goldman, Hodgkin and Katz current model (Hille 2001):

$$
I_{X}=N p_{o} P_{X} z_{X}^{2} \frac{F^{2} V_{m}}{R T} \frac{\gamma_{i}[X]_{i}-\gamma_{o}[X]_{o} \exp \left(-z_{X} F V_{m} / R T\right)}{1-\exp \left(-z_{X} F V_{m} / R T\right)}\left(V_{m}-E_{X}\right)
$$

with the membrane permeability $\mathrm{P}_{\mathrm{X}}$, the ion valence $\mathrm{z}_{\mathrm{X}}$, the intracellular and extracellular ion concentration $[\mathrm{X}]_{\mathrm{i}}$ and $[\mathrm{X}]_{\mathrm{o}}$, respectively, with the corresponding partition coefficients $\gamma_{i}$ and $\gamma_{0}$, the temperature $T$, the gas constant $R$, and the Faraday constant $F$.

A further way to describe the current through an ion channel is to consider the energy barrier of the channel pore. A pore has one or more internal binding sites at which the ion has to bind. A common approximation of the energy barrier approach is given by (Hille 2001):

$$
I_{X}=N p_{o} \frac{g_{X}}{1+\lambda \exp \left(b V_{m}\right)}\left(V_{m}-E_{X}\right)
$$

with the constants $b$ and $\lambda$ defining the voltage sensitivity and gradient of the barrier function respectively. This assumes that the pore is immediately in a steady state.

\subsection{Models of Ion Pumps and Exchangers}

Ion pumps are often described in terms of binding processes, where the transported ion binds to the transporter protein. The current $I_{p}$ in the kinetic models of the ion pump has the characteristics (DeFelice 1997):

$$
I_{p}=N I_{p, X, \max }\left(V_{m}\right) \prod P_{\text {sites }, i}
$$

with $\mathrm{I}_{\mathrm{p}, \mathrm{X}, \max }$ as the maximal current through the transporter and the probability of having occupied sites $\mathrm{P}_{\text {sites }}$ described by Hille (2001):

$$
P_{\text {sites }}=\frac{1}{1+\left(K_{m, X} /[X]\right)^{n}}
$$

with the equilibrium constant of the reaction $\mathrm{K}_{\mathrm{m}, \mathrm{X}}$ of ion $\mathrm{X}$ describing the concentration at half saturation, the concentration of the ion $[\mathrm{X}]$ and the number $\mathrm{n}$ of ions that can bind to the receptor.

Ionic exchangers use the energy available in one given ion's electrochemical gradient to transport another ion against its gradient. Since ionic exchangers can work in both forward and backward mode, the total exchanger current $I_{x}$ is given by the difference in the currents for the forward reaction $\mathrm{I}_{\mathrm{x}, \text { forw }}$ and for the backward reaction $\mathrm{I}_{\mathrm{x}, \text { back }}$ (Hille 2001):

$I_{x}=I_{x, \text { forw }}-I_{x, \text { back }}$ 
Both current components follow a description of binding processes. Since two ion types, each with two binding sites are involved, the current components are:

$$
I_{x, \mu}=N F k_{\mu}(V) P_{\text {sites }, X_{o}} P_{\text {sites }, Y_{i}}\left(1-P_{\text {sites }, X_{i}}\right)\left(1-P_{\text {sites }, Y_{o}}\right)
$$

with $\mu$ being either forward or backward mode, the forward and backward reaction rate $\mathrm{k}_{\text {forw }}$ and $\mathrm{k}_{\text {back}}$, respectively, and the probabilities of having occupied entrance sites $\mathrm{P}_{\text {sites }}$ for the ion types $\mathrm{X}$ and $\mathrm{Y}$ for either intracellular $i$ or extracellular $o$ binding sites. A simplification to this equation can be made at low concentrations by neglecting the saturation effect of one ion type or by assuming a balance relation between the forward and backward reactions, leading to only one reaction rate.

More detailed kinetic schemes for transporters involve description of the full enzymatic cycle and transition rates between states of the transporter. Ion pumps, in which the free energy of ATP hydrolysis is coupled to ion movement, are similarly described, and are constrained by thermodynamic considerations on the free energy transduction between ion transport and ATP hydrolysis. Such models may be simplified on the basis of rapid binding assumptions and steady state approximations where appropriate (Smith and Crampin 2004).

\section{Review of Existing Models}

Electrophysiological cell models integrate descriptions of ion fluxes through various ion channels, transporters and exchangers, to describe cellular properties such as transients in transmembrane voltage (the action potential) and intracellular calcium. Data sources for the development of cell models and their components include electrophysiological studies of ion channels and isolated cells as well as imaging studies of structure and function at molecular, cellular and sub-cellular scales. Models of cardiac cell function have focused on the key physiological processes underlying generation of the action potential and calcium signalling leading to contraction. Other aspects of cell function, such as key regulatory signalling pathways (including $\alpha$ - and $\beta$-adrenergic signalling pathways: Cooling et al. 2007; Saucerman et al. 2003), and cellular energy metabolism have also received considerable attention, but will not be addressed in detail here. Although many of the most basic biophysical mechanisms underlying electrophysiology, calcium handling and force development of cardiac cells are largely understood, models developed to study these functions vary significantly in complexity and their level of abstraction. Furthermore, although the field is making rapid progress in detailed identification and characterisation of the basic components involved in these functions, there remain significant differences between models developed to explain ostensibly the same set of phenomena. In this section we illustrate this issue with an introduction to a wide range of detailed models of the electrophysiology of mammalian cardiac cells, followed by a more detailed discussion of human ventricular cells. We will also introduce models of cell contraction and calcium signalling. These models exhibit a large diversity in their approaches to describing cellular properties. Finally, in this section we discuss approaches for model reduction.

\subsection{Overview of Mammalian Electrophysiology Models}


Many models of cell electrophysiology for different species and regions of the heart have been developed over the last several decades (for a review, see Fenton and Cherry (2008)). Along with human ventricular models, which are discussed in detail below, ventricular models have been developed for guinea pig (Luo and Rudy 1991, Nordin 1993, Luo and Rudy 1994, Noble et al. 1998, Faber and Rudy 2000, Matsuoka et al. 2003), canine (Winslow et al. 1999, Fox et al. 2002, Greenstein and Winslow 2002, Cabo and Boyden 2003, Hund and Rudy 2004, Flaim et al. 2006), rabbit (Puglisi and Bers 2001, Shannon et al. 2004, Mahajan et al. 2008), rat (Pandit et al. 2001), and mouse (Bondarenko et al. 2004, Wang and Sobie 2008). The existence of species-specific ventricular models is a significant improvement over earlier, more generic ventricular models and recognises the differences in current contributions across species. However, despite these advances, having multiple models for the same species can lead to confusion over cellular properties and mechanisms.

For example, the Fox et al. and Hund-Rudy canine models include many of the same currents, but, as shown in Figure 1, these currents often behave quite differently in the two models. The sodium-calcium exchanger current operates in reverse mode through much of the action potential plateau in the Hund-Rudy model, but not in the Fox et al. model. The magnitude of $\mathrm{I}_{\mathrm{Ks}}$ in the Fox et al. model makes its role during early and late repolarisation more significant than in the Hund-Rudy model, where the current is nearly an order of magnitude smaller. In the Hund-Rudy model, $\mathrm{I}_{\mathrm{K} 1}$ is not active during the plateau, whereas it is for the Fox et al. model; similarly, $\mathrm{I}_{\mathrm{Kr}}$ is significant primarily during repolarisation for the Hund-Rudy model, whereas it is also active during the plateau for the Fox et al. model. These differences in currents can lead to differences in model predictions (Cherry and Fenton 2007). For instance, as shown in Figure 1, although both the Fox et al. and Hund-Rudy models exhibit alternans at fast pacing rates, the Fox et al. model predicts that calcium transients are associated with every beat and that the magnitude of $I_{\text {to }}$ does not vary beat-to-beat, whereas the Hund-Rudy model predicts that calcium release and thus the calcium transient is blocked for the short action potentials and that the magnitude of $\mathrm{I}_{\mathrm{to}}$ varies with action potential duration.

\section{[figure 1 around here]}

Along with ventricular models, models of atrial cell electrophysiology have also been developed for different mammalian species, including rabbit (Hilgemann and Noble 1987, Lindblad et al. 1996), human (Nygren et al. 1998, Courtemanche et al. 1998), and canine (Ramirez et al. 2000). Purkinje models also have been developed, including both generic models (Noble 1962, McAllister et al. 1975, DiFrancesco and Noble 1985) and, more recently, human-specific (Stewart et al. 2009) and canine-specific (Aslanidi et al. 2009) models based on human and canine ventricular cell models, respectively.

Unlike atrial and ventricular models, sinoatrial node (SAN) models must be autooscillatory to produce spontaneous and rhythmic action potentials. Several mathematical models of the SAN action potential (AP) have been developed for mammalian species, including human (Chandler et al. 2009), rabbit (Noble and Noble 1984, Demir et al. 1994, Dokos et al. 1996, Zhang et al. 2000, Boyett et al. 2001, Kurata et al. 2002) and 
guinea pig (Sarai et al. 2003). Comparing the dynamical behaviour of various SAN cell models, in particular the functional role of each individual ionic current in generating pacemaking APs, it can be seen that these models show consistency in some respects, but diversity in others. In all cases, SAN models are able to reproduce periodic and autorhythmic action potentials and the experimentally observed depressive modulations by the surrounding atrium due to electrotonic interactions. However, models have implemented different subsets of ion channel currents, reflecting the limitations of experimental data and knowledge about SAN electrical properties at the time when these models were developed. As a consequence, the functional role of each individual current in these models may be different. Even in the most recently developed models - such as those by Zhang et al. (2000) and Kurata et al. (2002) discussed below-although the functional role of some major ionic currents is qualitatively similar, significant quantitative differences remain, as shown by differences in the sensitivities of the two models to model parameters (Kharche et al. 2009).

While SAN modelling has a lengthy history (Wilders et al. 1991, Demir et al. 1994, Dokos et al. 1996, Wilders 2007), this review will focus on recently introduced SAN models. Zhang et al. (2000) developed a family of SAN cell models that successfully reproduce the differences in the electrical properties of 'central' and 'peripheral' SAN cells. Recent advances in experimental electrophysiology have determined the electrical heterogeneity across the SAN and the surrounding atrium - cells in the SAN present pronounced regional differences in their electrical APs and associated properties of ion channels (Boyett et al. 2000). Experimental data obtained from different regions of the SAN show gradient variations of electrical APs from the centre to the periphery of the SAN. Correlations between current density and cell size for several ionic currents have been reported that match the correlation between the characteristics of APs and the cell size (Boyett et al. 2000).

At the same time, based on previous SAN models (Wilders et al. 1991, Demir at al. 1994, Dokos at al. 1996, Zhang et al. 2000), Kurata et al. (2002) developed an improved model for a primary SAN pacemaking cell. This model presented new formulations for the voltage- and calcium-dependent inactivation kinetics of $\mathrm{I}_{\mathrm{CaL}}$ and intracellular calcium regulation and buffering. Later on, this Kurata et al. model was modified to account for the heterogeneous properties of the SAN (Kurata et al. 2008), following the approach of the Zhang et al. (2000) model. Obviously, model development for the electrical AP of SAN cells has been an evolutionary process, which, like the previously described development of ventricular cell models, closely follows experimental progress. With increasingly advanced experimental techniques emerging, more detailed electrical properties for the SAN will be unveiled, warranting development of new or improved models for the SAN electrical action potential in the future.

\subsection{Electrophysiology Models of Human Ventricular Myocytes}

Recently, several models have been developed to describe the origins and form of the human ventricular myocyte action potential, an important step towards development of multiscale models of the human heart, and the potential for patient-specific modelling, one of the major ambitions of computational modelling of the heart and the physiome in 
general. Modelling of human ventricular cells started with a study by Priebe and Beuckelmann (1998), aimed at understanding the effects of electrophysiological alterations in heart failure. In this study, models were developed to describe normal and failing human myocytes with similar approaches to those previously used for cardiac cells of other species. The basis for their modelling was the Luo-Rudy phase-2 model of guinea pig ventricular myocytes, which was parameterised with data including transients of transmembrane voltage, ion fluxes and concentrations measured in normal and diseased human myocytes. A major achievement of this modelling study was the identification of relationships between disease-associated alterations at the level of transmembrane proteins and features of the action potential of human myocytes. More recent applications include integration with models of cellular contraction and tissue electrophysiology (Sachse et al. 2003, Niederer and Smith 2007, Niederer and Smith 2009).

In 2004, two further models of human ventricular cells were introduced, the Iyer et al. (2004) and ten Tusscher et al. (2004) models. Both provide significantly more detail on ion channel function and compartmentalisation of ion handing within the cell. The increase in detail (and complexity) reflects new insights in channel function and the availability of novel measurement data from human cells and ion channels. The Iyer et al. model describes the electrophysiology of subepicardial cells, applying Markovian models for most channels. In contrast, ten Tusscher et al. modelled subendocardial, midwall and subepicardial cells, commonly using Hodgkin-Huxley type models. A revision of this model, published in 2006, includes more detail on intracellular calcium handling. Detailed comparisons of these human ventricular models, including ion currents, action potential shapes and durations, rate adaptation, and other properties, have been performed to assess the similarities and differences of the models (ten Tusscher et al. 2006, BuenoOrovio et al. 2008).

The Priebe and Beuckelmann (1998), Iyer et al. (2004) and ten Tusscher et al. (2006) models exhibit significant differences in the reconstruction of transmembrane voltages, ion currents and concentration during an action potential. These differences can partially be explained by the differences in the experimental data used to characterise these models (Niederer et al. 2009), the use of different model structures and quality of fit.

Figure 2 demonstrates the disparity in model predictions for the membrane potential, calcium transient and $\mathrm{I}_{\mathrm{Kr}}$ channel current. The significant differences in these metrics of cardiac function have ramifications for the application and reuse of these models. Specifically the different action potential morphologies and $\mathrm{Ca}^{2+}$ transients will cause significant changes in the predicted conduction velocities in tissue scale models and active tension generation in electromechanics models. In silico studies of drug interactions and mutations may show effects that are model-dependent. Experiments where these models are embedded in tissue simulations may have different conclusions, and linking these models to models of contraction will have a major impact on the resulting tension transient. While it is important to recognise that some differences between models are to be expected given biological variability and the scarcity of human 
data, the problem arising with the current models is that the source of the variability is poorly characterised, hindering appropriate reuse and continued development.

[Figure 2 about here.]

\subsection{Models of Cell Contraction}

The modelling frameworks described above outline the components of cardiac electrophysiology models. While the development of computational cell-based representations of cardiac contraction has paralleled cardiac electrophysiology developments, these models have, in general, lagged in complexity. More recently, detailed biophysical models of the inner workings of the sarcomere have emerged. These models are now capable of facilitating the investigation of the mechanisms responsible for relaxation (Niederer et al. 2006), heterogeneous tension development (Campbell et al. 2009) and cooperative activation (Rice et al. 1999).

Early models of contraction aimed to characterise the biochemical states of the cross bridge (Eisenberg and Moos 1968, Lymn and Taylor 1971, Stein et al. 1979) or characterise tension development in the absence of thin filament regulation (Huxley and Simmons 1971). More recent models have aimed to capture both thick and thin filament kinetics (Razumova et al. 1999, Rice et al. 1999, Sachse et al. 2003, Niederer et al. 2006, Schneider et al. 2006, Rice et al. 2008). In most cases these models are represented by systems of ordinary differential equations, and potential spatial effects are included in phenomenological representations or are ignored. Models that aim to capture spatial information often require the solution of computationally more expensive partial differential equations (Huxley 1957), cellular automata (Zou and Phillips 1994, Dobrunz et al. 1995) or Monte Carlo simulations (Hill et al. 1981, Daniel et al. 1998, Rice and de Tombe 2004). This additional complexity allows these models to capture important phenomena such as filament compliance, protein-protein interactions and cross bridge distortion. All of these mathematical approaches for modelling contraction require extensive data. However, the necessary experimental measurements are often incomplete in cardiac tissue or are for a specific species or temperature, limiting the capacity to robustly combine the data into a common quantitative framework for cardiac tension development.

The scarcity of experimental data for different species at physiological temperatures restricts the development of models and limits the confidence that model conclusions are correct or relevant at $37^{\circ} \mathrm{C}$. Models can either aim to simulate contraction at room temperature (Niederer et al. 2006), scale parameters (Rice et al. 2008) using the temperature coefficient $\mathrm{Q}_{10}$ (Hille 2001), or ignore temperature effects altogether. More recently experimental measurements of contraction are becoming available at $37^{\circ} \mathrm{C}$ in trabeculae (Varian et al. 2006) and isolated single cell preparations (Iribe et al. 2007). This may reduce the difficulty of developing models of contraction at body temperature in the future.

Measurements at $37^{\circ} \mathrm{C}$ in ex vivo and in vivo preparations begin to allow the comparison of the development of tension across multiple spatial scales under physiological 
conditions. Unfortunately, reconciling measurements made at different spatial scales is nontrivial. At the single cell scale, measurements of isometric contractions in rat at $37^{\circ} \mathrm{C}$ record peak stress of $\sim 6 \mathrm{kPa}$ for physiological work loops (maximum sarcomere length $\sim 2.0 \mu \mathrm{m}$ ) (Iribe et al. 2007). This is in comparison to measurements of peak developed stress at $\sim 35 \mathrm{kPa}$ under isometric conditions in trabeculae (sarcomere length $2.2 \mu \mathrm{m}$ ) (Varian et al. 2006) and to calculations of in vivo stress of $\sim 25 \mathrm{kPa}$ in rat hearts (Holmes, 2004). Although these measurements are not designed to be equivalent, there is as yet no modelling-based explanation that can reconcile all three measurements.

As noted in the introduction, another issue encountered in combining and reusing components of models is the inheritance of model parameters over multiple generations of models, without transparent links to the data supporting these parameter value choices. This lack of model provenance inevitably results in the development of mathematical models built on measurements which have since been superseded due to improvements in measurement techniques. In cardiac cell modelling this phenomenon can be demonstrated in a number of parameters. In particular, the parameter values describing the unbinding of calcium from troponin $\mathrm{C}$ are routinely taken from data recorded thirty years ago which have since been superseded but have been inherited by recently developed models from earlier ones (Smith et al. 2007). In a recent review, the dissociation rates of calcium were listed as $42 \mathrm{~s}^{-1}, 105 \mathrm{~s}^{-1}$ and $13 \mathrm{~s}^{-1}$ for isolated troponin, troponin contained within the thin filament and troponin in the thin filament in the presence of bound cross bridges, respectively (Davis and Tikunova 2008). Cardiac electrophysiology models often do not include tension development, but given that peak tension is reached during the decay phase of the calcium transient and that calcium buffering will have the greatest effect during peak calcium, the static unbinding rate of calcium could be approximated as $\sim 100 \mathrm{~s}^{-1}$. This is not easily compatible with the routinely used values of $19.6 \mathrm{~s}^{-1}$ (Bondarenko et al. 2004, Mahajan et al. 2008, Shannon et al. 2000), 40 $\mathrm{s}^{-1}$ (Iyer et al. 2004, Pandit et al. 2001, Winslow et al. 1999) and 200s ${ }^{-1}$ (Noble et al. 1998).

\subsection{Models of Calcium Signalling in Cardiac Cells}

The modelling of calcium as one of the most important second messengers spans a wide range of modelling approaches, the majority of which seek to provide insight into two important questions. Firstly, how does the cellular release and uptake system for calcium shape the intracellular calcium concentration, and secondly, what is the downstream effect of a given calcium signal? The dominant pathways for calcium to enter the cytosol are through voltage-gated L-type calcium channels from the extracellular space and through RyR and $\mathrm{IP}_{3} \mathrm{R}$ channels from the SR (Bers 2008). In the opposite direction, plasma membrane calcium ATPase (PMCA) pumps and sodium-calcium exchangers (NCXs) transport calcium from the cytosol into the extracellular medium, while sarcoendoplasmic reticulum calcium ATPase (SERCA) pumps resequester calcium to the SR. Note that depending on the experiment that the model addresses, not all of these calcium transport mechanisms need to be represented. For example, pathways involving the SR may be omitted in a study of inhibited luminal dynamics (Michailova et al. 2002), and plasma membrane processes can be excluded if the emphasis lies on spark generation and statistics (Nguyen et al. 2005, Hinch 2004). The degree of detail to which ion channels, transporters and pumps are described is tightly coupled to their numbers as well as to the 
size of the spatial domain under investigation. If both are small, Markov chain models as outlined in Section 3.1 can provide very detailed insight into biophysical mechanisms. These models can still be used when coupled to a larger cell section provided that the number of calcium handling molecules is still small. In this case, hybrid approaches as described, e.g. in Rüdiger et al. (2007) have proven fruitful. At the cellular level, the use of hybrid models is prohibitive due to their large computational cost, but a recent study using waiting times has illustrated how to analyse whole-cell calcium dynamics without the loss of information on the molecular scale (Thul et al. 2009).

A key feature of calcium release and uptake in myocytes is a prominent spatial heterogeneity. Liberation through RyR channels is restricted to discrete positions on the SR, and the coupling between L-type calcium channels and RyR channels occurs only at the dyadic cleft. Different modelling frameworks have been put forward to address this spatial inhomogeneity, most notably compartmentalised approaches and models based on partial differential equations (PDEs). In Section 4.4, we provide a more detailed discussion of these two modelling routes and highlight how calcium buffers, a dynamical SR and mitochondria shape intracellular calcium concentrations even further.

Spatio-temporal variations of the cytosolic calcium concentration act as input to a multitude of cellular responses such as contraction and hypertrophy (Berridge et al. 2000). Both processes present prime examples for the distributed character of calcium signalling. They are initiated at the plasma membrane, but the final destinations are the myofilaments in the centre of the myocyte and the nucleus with its own calcium dynamics, respectively. Recent modelling approaches reflect the spatial set-up of these signalling cascades by employing both compartmentalised and PDE models (Cooling et al. 2009, Korhonen et al. 2009, Cooling et al. 2007).

A prominent feature of intracellular calcium dynamics is the occurrence of complex spatio-temporal patterns (Wier et al. 1987), ranging from localised elevations of the cytosolic calcium concentration over propagating waves to whole-cell transients (Berridge et al. 2000). For cardiac cells, it was recognised early on that the cellular morphology considerably shapes the temporal evolution of calcium concentration profiles (Soeller et al. 2009). However, model design decisions such as the choice of sub-cellular structures and the degree of biophysical detail to be included in cardiac calcium models has given rise to a broad variety of modelling approaches. Nevertheless, a principal division can be made between lumped models and spatially distributed approaches in the form of partial differential equations. In the first case, compartments represent functional intracellular elements, e.g. the bulk cytosolic calcium concentration corresponds to one entity that has certain influxes and effluxes whose time constants are related to diffusive properties. In the second group of models, the intracellular space is explicitly resolved, which allows, e.g. for an exact treatment of diffusive transport. Although the latter approach offers the advantage of including sub-cellular detail, it comes at a significantly higher computational cost in comparison to lumped models.

The compartmental approach was first implemented by DiFrancesco and Noble (1985) in a Purkinje cell model that for the first time described intracellular calcium dynamics 
including separate pools for cytosolic, non-junctional SR (NSR) and junctional SR (JSR) calcium concentration. From the simplest approach representing only cytosolic and SR compartments (Fox et al. 2002), the transition to the most complex model with cytosolic, junctional, subsarcolemmal, NSR, JSR and troponin compartments (Mahajan et al. 2008) is not obvious, since a number of "intermediate" approaches exist. Table 1 highlights this variety further, where we group models according to the number of compartments represented. The justification of separate compartments for the cytosolic, junctional and subsarcolemmal space arises from experimental findings that during a normal action potential cycle, the calcium concentration in the vicinity of calcium release sites reaches peak values 10-100 times (Langer and Peskoff 1996) and, close to the sarcolemma, values 2-3 times (Weber et al. 2002) higher than in the cytosol. The division of the SR into two compartments, network and junctional, is employed to represent the experimental observations that close to the calcium release junction there are parts of the SR that are depleted of calcium during each release to a much larger extent than the SR in other areas of the cell (Shannon et al. 2003). That is, the junctional SR compartment refers to a releasable calcium pool, which is "separated" from the network SR due to a significant diffusion resistance (Wussling and Szymanski 1986).

[Table 1 around here]

A mitochondrial calcium compartment is typically omitted from cardiomyocyte models. This omission generally has been justified by findings that the role of mitochondria as a calcium sink or source in beat-to-beat regulation of the cytosolic calcium concentration is rather small (Bassani et al. 1992). However, recent results from various groups suggest that mitochondrial calcium uptake and release can have a significant effect on cellular calcium homeostasis (Duchen et al. 1998, Trollinger et al. 2000), see Dedkova and Blatter (2008) for a review. Thus, it appears that the addition of a mitochondrial calcium compartment should be addressed in future modelling studies. This intention is supported by recent developments in the mathematical description of mitochondrial calcium dynamics (Nguyen et al. 2007, Dash et al. 2009). Most recently Pásek et al. (Pásek et al 2008a, Pásek et al 2008b) have developed compartmental models that explicitly include the transverse-axial tubular system. These models have been applied to assess the importance of ion channel heterogeneity and restricted diffusion within the tubular system on physiological function.

The fundamental challenge of the compartmental approach is the lack of corresponding distinct anatomical structures inside cells. Thus, there is no straightforward way of choosing the appropriate selection of compartments for a specific research question. Furthermore, model validation always requires that these compartmental parameters are derived from experimental data by some procedure, rather than by direct comparison with measured data.

One of the motivations in developing models with an increasing number of compartments was the insight that the calcium concentration in originally unrefined compartments was not as spatially homogeneous as initially assumed. Although this approach could be continued and more compartments introduced to account for a larger degree of cellular 
heterogeneity, an alternative approach is to consider space- and time-dependent concentrations within a single spatial domain. The calcium concentration then exhibits large spatial gradients around release sites upon calcium release, and decays to resting conditions in quiescent cells. As with the compartmentalised models, the complexity of partial differential equation-based models for cardiac calcium dynamics has increased over the years. Early works focused on a one-dimensional representation of the cytosol and were instrumental in our understanding of calcium waves in cardiac myocytes (Dupont and Goldbeter 1994, Pearson and Ponce-Dawson 1998, Keizer et al. 1998, Dawson et al. 1999). More recently, these models were extended to incorporate stochastic release and a dynamic SR (Coombes and Timofeeva 2003, Coombes et al. 2004, Thul et al. 2008). While integrating additional elements of calcium signalling into cardiac models has been of ongoing interest, a parallel development has been focusing on the implementation of realistic cellular geometries. This has resulted in three-dimensional simulations of calcium concentration profiles and has provided the first insights into the coupling between cell shape, ion channel and receptor distributions, and calcium signaling (Izu et al. 2001, 2006, Michailova et al. 2002, Means et al. 2006, Li et al. 2007, Li and Holden 2009, Korhonen et al. 2009, Lu et al. 2009; and see Lemon 2004 for an analytical study). Although consensus is emerging as regarding core components of any cardiac calcium model, details of some key mechanisms are still debated. There is no doubt that the dynamics in the SR plays a vital role, but some studies report fast luminal calcium diffusion (Wu and Bers 2006), whereas others support a small diffusion coefficient (Swietach et al. 2008). However, different ion mobility has far-reaching consequences for wave propagation. Measurements of the geometry of the SR have revealed a highly tortuous structure, which in turn reduces the effective velocity of diffusional transport. Whether models need to explicitly incorporate the shape of the SR or whether an effective reduction of the diffusion coefficient as suggested by Olveczky and Verkman (1998) suffices is still an open question. The impact of mobile and stationary buffers on calcium signals, both in the myoplasm and the SR, is now firmly established. Specifically, key properties such as the buffering capacity still lack final quantification (Trafford et al. 1999, Walden et al. 2009), and the regulatory role of buffers such as calsequestrin has emerged only recently (Györke et al. 2004, Chopra et al. 2007, Györke and Terentyev 2008). Furthermore, as the calcium-sensitive fluorescent dyes added during experiments to measure calcium concentration are themselves exogenous calcium buffers, careful analysis (often using models) of the measured fluorescence trace is required to quantify the calcium transient from the raw data. The variety of cardiac calcium models reflects the uncertainties that we still face with respect to both underlying mechanisms and parameter values, but continuous advances in theoretical and experimental techniques will promote the convergence of all models towards a unified framework of cardiac calcium signalling.

\subsection{Reduced Electrophysiology Models}

To study cellular properties at a higher level than the specific formulations of individual ion channels, such as rate adaptation, memory and bifurcations, less biophysically detailed models also can be useful. Of course, one of the most important advantages of reduced models is their increased computational tractability, which can permit larger, longer and more spatially resolved three-dimensional simulations. As discussed below, 
there are three different approaches for reducing model complexity: simplified models, minimal models and discrete iterative maps.

Simplified models are generally focused on using the time scales associated with different processes represented in the model to reformulate the model equations. Gating variables with fast time constants, for instance, can be adiabatically eliminated and replaced with their steady-state functions, thereby reducing the number of equations. For example, the two-variable Karma model (Karma 1993) is a simplification of the fourvariable Noble model (Noble 1962), with the $\mathrm{m}$ and $\mathrm{h}$ gates eliminated in this manner. In addition, some gating variables with slow time constants may not vary enough to affect the model behaviour significantly and can be eliminated; similarly, currents with very small amplitudes in some cases also can be eliminated without significantly affecting most cellular properties. The Bernus et al. (2002) reduction of the Priebe-Beuckelmann human ventricular model is an example of a reduced model that obtains good agreement with the original model, as shown in Figure 3. In this case, the number of variables was reduced from 17 to six by eliminating four of the original nine gating variables and by treating all ionic concentrations as constants.

\section{[figure 3 around here]}

Minimal models reflect the minimum number of equations required to reproduce the global dynamics of the transmembrane voltage without explicitly representing each individual transmembrane current (Fenton and Karma 1998, Bueno-Orovio et al. 2008, Biktasheva et al. 2006). In general, three state variables allow sufficient flexibility to fit important cellular properties, including the threshold of excitability, upstroke rate of rise, action potential duration (APD) and amplitude, and the APD restitution function, as well as conduction velocity (CV) and the CV restitution relation in tissue. However, to reproduce the morphology of spike-and-dome action potentials, an additional variable is required (Cherry and Fenton 2004, Bueno-Orovio et al. 2008). These models can be obtained by asymptotic approaches different from the standard fast-slow reductions used in simplified models (Biktashev et al. 2008).

Because simplified and minimal models do not include detailed biophysical descriptions of most cellular process, their application in, e.g. studies of specific effects like ion channel blockade or mutations, is less straightforward.. These models are intended primarily for use in tissue simulations where multicellular effects are being investigated and computational efficiency becomes important. In some cases, simplified or minimal models can be used to represent the overall membrane dynamics under various conditions, including pharmaceutical agents or mutations, by fitting model parameters directly from experimental data, a process that becomes faster and more straightforward when the number of parameters is reduced significantly (often by an order of magnitude) (Bueno-Orovio et al. 2008, Bernus et al. 2002). These model variations then can be simulated in tissue to study tissue-level phenomena, including wave stability and dynamical bifurcations (see, for example, the paper in this issue). 
Discrete iterative maps are used to study certain characteristics of cell dynamics without tracking the membrane potential, gating variables, currents, and concentrations explicitly, but instead by developing and using equations to compute quantities like APD or peak calcium concentration as a function of these and other discrete quantities. They can be as simple as iterative functions that describe rate adaptation from standard experimental APD restitution curves (Nolasco and Dahlen 1968, Guevara et al. 1984) or from cell models (Cain and Schaeffer 2006, Schaeffer et al. 2007, 2008). Slightly more complex maps can be created to describe the dynamics of intracellular calcium alone (Eisner et al. 2000) or combined with the membrane potential (Qu et al. 2007) to study the dynamics and types of bifurcations during alternans.

\section{Model Identification-Model Selection}

Depending on the amount of available data and the knowledge of underlying mechanisms, the described models can be more or less complex. For example the fast sodium channel has been described using between two and 21 states and nine and 171 parameters (Hodgkin and Huxley 1952 and Kahlig et al. 2006, respectively). The necessary and sufficient complexity of the model design depends on the modelling goal. For example the information content in voltage-clamp steps for voltage-gated ion channels provides approximately 15 parameters (see Fink and Noble 2009). Figure 4 shows that the optimised voltage-clamp step protocol presented in Fink and Noble (2009) provides more information than a normal action potential clamp, indicating that it should be sufficient for a model to fit data from the optimised voltage clamp step protocol if the goal is to replicate whole cell phenomena.

[Figure 4 around here]

With regards to the computational aspects determined by the model design, it has been shown that stiffness is more stringent than the number of ordinary differential equations regarding the slowing down of single cell computations (e.g. Fink and Noble 2009). Stiffness can be eliminated by separation of time scales, for instance by asymptotic approximations (e.g. Hinch et al. 2004). Principal component analysis or sensitivity analysis can be used for removal of parameters or model parts to which the output is insensitive (e.g. Fink and Noble 2009, Bernus et al. 2002, Schmidt et al. 2008). Lately, a similar approach of invariant manifold reductions for Markov models has been used (Keener 2009).

The final decision concerning appropriate model design must be made using validation of the models using the full range of available experimental data outlined in Section 3. However, so far the general lack of ability to fit the parameter values has made it impossible to compare the validity of different model designs. This is because differences in their predictive power could have come from the framework of model states and transitions or from the quality of the parameter fit, e.g. the error between model output and experimental data.

\subsection{Parameterisation, Data Availability and Local Minima}


Experimental design can be used to determine the experimental protocols necessary for estimation of the model parameters. However, it still remains difficult to find the right amount of data for obtaining a full action potential model using data from single cell experiments. Boundaries on conductances and maximum rates can be given from experimental data, but to find the optimal parameter set, local identifiability of the parameters is necessary at least, whilst still avoiding overdetermination (Dokos and Lovell 2004).

Table 2 shows the parameter identifiability results using the ten Tusscher model (ten Tusscher and Panfilov 2006). The voltage traces for one action potential using patch clamp recordings would only provide 4 conductances (for $i_{\mathrm{Na}}, i_{\mathrm{CaL}}, i_{\mathrm{Kto}}$, and $i_{\mathrm{Kr}}$ or $i_{\mathrm{Ks}}$ ) out of 12 (neglecting background currents). Adding measurements of intracellular calcium for the same cell would provide only two more parameters (for NCX and SERCA). Having the complete data for a S1S2 protocol would provide eight parameters for voltage only and 10 for additional data on intracellular calcium. Only by measuring the amount of calcium in the dyadic cleft would all 12 parameters be obtained. Another possibility giving full identifiability would be to have voltage and intracellular calcium measurements for two different extracellular potassium concentrations (see Table 2)

[Table 2 around here]

In addition to the problem of finding the right amount of data, the nonlinearity of the minimisation problem and the associated numerical problems make finding an optimal fitting algorithm difficult. Furthermore, the number of local minima typically becomes larger and more problematic as model complexity is increased to include dozens of variables and hundreds of parameters. The availability of simple methods like the Simplex and Levenberg-Marquardt algorithms provided in Matlab ${ }^{\mathrm{TM}}$ or a recently developed CellML-based tool (Hui et al. 2007) and their limited need for significant computational resources would make them good candidates but their ability to find global minima is very restricted given the number of parameters to fit. Similar computational needs and problems with large numbers of parameters apply for the maximum likelihood method which is used for single channel measurements as in the software package QuB (Milescu et al. 2005). Sequential quadratic programming methods also can be used to find a constrained minimum with superlinear convergence (Gill 1981, Fletcher 1987) for model parameters using Matlab ${ }^{\mathrm{TM}}$ (Bueno-Orovio et al. 2008). Global methods like genetic algorithms, which have been used to fit conductance values (Syed et al. 2005), simulated annealing (e.g. Kirkpatrick et al. 1983) and particle swarm algorithms (e.g. Kennedy and Eberhart 1995) are perhaps more promising, but the amount of resources necessary is far higher than for other algorithms.

\subsection{Sensitivity of Parameters and Their Confidence Interval}

Statistical fitting algorithms (as used in QuB) immediately provide the confidence intervals of the parameter values. Once the "global" minimum is found using a deterministic algorithm with one specific data set, the confidence interval can be determined by using either the covariance matrix (Seber and Wild 2003) or more generally using statistical tools like bootstrap algorithms (Carpenter and Bithell 2000) 
and multivariable regression (Sobie 2009). For reasonably simple models one can determine parameter values, the confidence intervals and the "cell-to-cell variability" simultaneously using nonlinear mixed-effect models (e.g. Meza et al. 2007). If the sensitivity of the model output to a specific parameter is very high then the confidence interval will also be very large and a different model design might be preferable.

\subsection{Validation with Distinct Data, Identification of Limitations and Possible Extrapolation}

Due to the fact that, as discussed in the introduction, many models are fitted to a very broad selection of (sometimes inconsistent) experimental data, there is often no additional experimental data available to actually validate the model. Specifically, there may not be enough data to determine if the model design with the given parameters is only a good interpolation for the data used to fit the model, or if the model provides a good approximation of the biophysical system it is representing and therefore has the ability to be used for extrapolation.

With the ability to determine the amount of data actually needed to determine the parameters uniquely (as discussed above), remaining data sets under the same experimental conditions can be used to validate the ability of a model to replicate the behaviour of, for instance, the hERG channel for various voltage clamp steps, action potentials, or the dependence of the current response on extracellular potassium. Validation thus provides information on the limitations of the model and the range of protocols where the model has predictive power. As a model will provide incorrect results when used outside of its scope, the validation process represents one of the necessities for the success of the Physiome project, which is strongly based on model reuse.

As an example, data obtained at different temperatures can pose a challenge to constructing a realistic model. The experimental data used in developing a given model is often obtained over a range of temperatures and subsequently rescaled, although this rescaling has not been well established and is likely highly nonlinear. However, models generally do not incorporate the effects of temperature beyond its effect on Nernst potentials. For instance, microelectrode recordings show that action potentials recorded at different temperatures differ substantially; however, model action potentials often do not replicate this temperature dependence, as shown in Figure 5.

\section{[Figure 5 around here]}

As the complexity of mathematical models of cardiac myocytes continues to grow it becomes increasingly difficult for a single research group to fully redevelop a new model for their specific scientific question. This inevitably leads to the reuse and adaptation of previous models for applications outside the remit of the original model. The number of physiological phenomena captured by mathematical models is vast and continues to grow. It can often be challenging and time-consuming to determine the set of virtual experiments that should be performed on a model to evaluate if it is the best model for a new application. One possible solution is the development of standardised simulations 
that would allow a user to compare the characteristics of multiple models under a variety of unambiguous conditions. A number of models have been compared already in terms of their currents, concentrations, action potential morphology, rate adaptation and other properties, including models of human atrial (Nygren et al. 2001, Cherry et al. 2008), human ventricular (ten Tusscher et al. 2006, Bueno-Orovio et al. 2008) and canine ventricular (Cherry and Fenton 2007) cells. Table 3 shows calculated APD ${ }_{90}$ values from simulations for five species-specific models using three simple protocols. These simulations show how the model responds to changes in pacing frequency and extracellular calcium and potassium. It is noteworthy that not only do models show opposite changes in $\mathrm{APD}_{90}$ following changes in extracellular ions but also some models do not reach a stable limit cycle under certain conditions. Both these observations will influence the selection process of a model by a user and should be more readily available.

[Table 3 about here]

\section{Discussion}

Significant challenges in cardiac cell modelling remain in effectively identifying and applying appropriate and relevant data for the purposes of model development, identification, validation, refinement and extension. As illustrated above, model development and validation often fail to make reference to all of the available data relevant to processes captured by a given model. This issue is compounded by the fact that in adopting and extending established models, the original data set (and thus the proper context) on which a model is based is often lost or ignored (and thus the model context is corrupted). Moreover, as models grow more complex and diverse, we are increasingly faced with disagreements (in terms of their predicted qualitative or quantitative behaviour) between models purporting to simulate the same system (Nygren et al. 2001, ten Tusscher et al. 2006, Cherry and Fenton 2007, Wilders 2007, BuenoOrovio et al. 2008, Cherry et al. 2008, Cherry and Evans 2008). Assuming that disagreeing models are constructed with appropriate care and expertise, this phenomenon must be a consequence of disagreement in either the underlying data or the assumptions used to build the models. Ideally in such cases, models should provide a vehicle to elucidate and reconcile the source of apparent disagreement.

In response to these challenges, we propose a number of initiatives to help establish broader, deeper, and more transparent connections between models and experimental data than are typically associated with currently used practices. Specifically, we propose that the cardiac cell modelling community cooperate in: (1) applying state-of-the-art tools for model identification, sensitivity analysis and optimal design of experiments for model identification and parameterisation; (2) appropriately reconciling the complexity of models with the availability of data and the goals of a particular model; and (3) building a database of experimental data associated with cardiac cell modelling.

\section{Initiative 1: Application of Tools for Optimal Model Identification}

Identification of a complex model (that is, sensitive and reliable determination of appropriate parameter values) and validation of the basic model framework require comparison to a rich set of experimental data. In essence, it is necessary to design proper 
experimental protocols and optimal data sampling schedules for minimising the error and covariance in parameter estimates (Banga and Balso-Canto 2008). Such design requires initial models to simulate potential experiments, which should be designed to refine the models. Thus the process requires not only iteration between experiment and simulation, but coordination in using models to formally design the best possible experiments and using experiments to formally identify the best possible models.

Adopting optimal design approaches will require the development of appropriate software to facilitate the process. There exist numerous software environments for model simulation, such as COR and PCEnv (Garny et al. 2008), some of which incorporate tools for parameter estimation and sensitivity analysis. For example, the JSim package incorporates sophisticated optimisation routines for parameter estimation as well as the ability to automatically calculate sensitivity functions and generate multiple simulations by looping through ranges of parameter values. The QuB (Milescu et al. 2005) and Neuron (Brette et al. 2007) packages provide the ability to simulate and fit models of ion channels or cells. Yet none of these packages provide the means to systematically interrogate parameter identifiability. Furthermore, few tools exist for designing experiments for optimal model identification. Existing tools for optimal experimental design are targeted to pharmacokinetic experiments and simulation (Foracchia et al., 2004) and not easily ported to cardiac cell modelling and experiments.

Thus the opportunity (and need) exists for much progress in developing tools in this area. The approaches outlined in Section 5 of this paper will become part of the standard tool set of the cardiac cell physiology community only when easy-to-use and reliable software for experimental design, parameter estimation and analysis of sensitivity and covariance is available.

\section{Initiative 2: Reconciling Model Complexity with Data Availability and Modelling Goals}

Models with more degrees of freedom typically provide closer fits to data, but at the expense of the ability to predict (or be validated against) new data. Model complexity is also at issue when relating the level of detail included in models to the goals of the modelling study. The problem of reconciling model complexity with the data available to parameterise the model and with the desired purpose of the model development is rarely directly addressed by the Physiome modelling community. Demonstration of appropriateness of model complexity and parameterisation should be a key requirement in the modelling process.

An advantage of biophysical modelling, wherein the mathematical description of a kinetic scheme or other process is constrained by known biophysical, chemical, structural or thermodynamic considerations, is that it restricts the form that a model for a given biological process may take. Tools and algorithms are available to assist with modelling within this paradigm (see, for example software available under the CellML ${ }^{2}, \mathrm{SBML}^{3}$ and

\footnotetext{
${ }^{2} \mathrm{http}: / /$ www.cellml.org/tools

${ }^{3} \mathrm{http}: / /$ sbml.org/SBML_Software_Guide
} 
Virtual Cell ${ }^{4}$ community websites), including algorithms to implement the King-AltmanHill approach to deriving steady state rate equations for biochemical cycles and for deriving and simulating multi-compartment multi-species biochemical reaction systems (Cornish-Bowden 1977, Qi et al. 2009, Vanlier et al. 2009).

Models developed using such approaches may contain a large number of parameters. Standard approaches for model reduction are based around simplifying assumptions such as rapid equilibrium steps in which specific reactions or binding-unbinding steps are assumed to take place on a faster timescale than other cellular processes of interest (see, e.g. Tran et al. 2009), or quasi-steady state assumptions (classically used in the derivation of the Michaelis-Menten expression for the rate of enzyme-catalysed reactions). Such simplification strategies produce models with fewer degrees of freedom, but which retain the underlying biophysical and thermodynamic principles on which they were constructed (Smith and Crampin 2004).

Different modelling assumptions, simplification strategies or alternate views on mechanism give rise to alternative models for a given cellular process. Selection between such competing models can be made on the basis of a parsimony principle (see, e.g. Burnham and Anderson 2002); formalised in approaches such as Akaike's Information Criterion (Akaike 1974), and Rissanen's Minimum Description Length (Rissanen 1978). Other methods which are also proving very useful in this area include Markov-chain Monte Carlo (MCMC) methods, using, for example, single channel data to determine model structure and parameterisation (e.g. Gin et al. 2009).

One further approach useful here is in assessing parameter sensitivity - that is, on which parameters in a model is the behaviour of interest strongly dependent. Measures of global parameter sensitivity based on sampling of local sensitivities (for example the Morris method: see Cooling et al. 2007) can be useful in indicating those parameters which are most important to determine experimentally. We note, however, that in a recent analysis of parameter sensitivity in models of a range of biological networks Gutenkunst et al. (2007) identified parameter 'sloppiness'-implying that despite being ill-constrained, the precise determination of at least some parameters may not be essential, while the behaviour of the system depends only on a few 'stiff' parameter combinations.

Thus the aims of the Physiome initiative, including model reuse, are best served when models of appropriate complexity (suitable for their intended purpose) are developed in line with biophysical principles, with parameter identifiably and appropriate parameterisation considered. We argue that the onus should be on authors of models to demonstrate that a model is not overfitted for its intended purpose, during the publication process.

\section{Initiative 3: Establishment of a Repository of Experimental Data Connected with Models}

As models grow in sophistication and complexity, it is necessary that the scope of the data on which they rest grows commensurately. Yet the ability to revise, reuse, extend

\footnotetext{
${ }^{4}$ http://vcell.org/
} 
and combine established cardiac cell models has rapidly outpaced the access to the original data on which cardiac cell models are based. Thus we propose to develop an open community-driven cardiac cell physiology data repository.

There exist a number of well established repositories for experimentally obtained data. Notably, the Worldwide Protein Databank (which grew out of the Protein Databank, established in 1971 at Brookhaven National Laboratory), is an international resource to archive and disseminate biomolecular structure data (Berman et al. 2003). Similarly, public repositories exist for gene sequence data (e.g. the NCBI Genome database), RNA microarray data (e.g. ArrayExpress (Brazma et al. 2003), Gene Expression Omnibus (Edgar et al. 2002) and the Center for Information Biology Gene Expression Database (Ikeo et al. 2003)) and proteomic data (e.g. PRIDE, PeptideAtlas, Global Proteome Machine Database, Tranche and Human Proteinpedia).

While for sequence and structural data the possible formats are relatively simple and straightforward to standardise, RNA expression and proteomic data cannot be separated from the experimental and measurement context. Thus as we move up in scale and complexity, the databases are necessarily more sophisticated in terms of their scope and flexibility. The RNA and proteomic database/repository efforts rely on minimal information standards MIAME (Brazma et al. 2001) and MIAPE (Taylor et al. 2007), which help to define the scope of what information is required to unambiguously describe a given dataset.

It would also be highly desirable to include experimental data at the cellular level that would include detailed information about cardiac action potentials over a broad range of stimulation rates. Such information could include the rate dependence of resting membrane potential, action potential duration, action potential morphology, action potential amplitude, upstroke velocity $\mathrm{dV} / \mathrm{dt}_{\max }$, and the threshold of excitation for both isolated cells and single cells in multicellular preparations. Detailed information on these properties could provide valuable constraints and additional validation data for cell models (Cherry and Fenton 2007, Bueno-Orovio et al. 2008).

It is not practically possible to develop an analogous set of standards to cover the breadth of cardiac cell physiology experiments. Yet this fact should not justify a failure to move forward on developing an open repository for widely disseminating data for use in any number of research applications, including model development and analysis. Only when the original data are available will it be possible to properly validate model components reused and/or extended for new applications. Such a community-driven data repository would provide a means for preserving associated data along with cardiac cell models. Furthermore, it would help make conflicts between data sets and conflicts between models and data apparent and transparent, inspiring further investigations and progress on resolving the conflicts. Thus we believe that such an open data repository will be a crucial resource for the field. It is inevitable that such an initiative must have the engagement of, if not also be driven by, the experimental community, who will be required to populate such a repository. To provide scientific incentive for such curation work the modelling community must, in turn, provide the tools necessary to gain new 
insights not possible from intuitive analysis of the data alone. It is meeting this challenge that will be fundamental to the success of the Physiome vision in the area of cardiac cellular modelling in the future.

\section{Acknowledgements}

This manuscript is the product of discussions at the Cardiac Physiome Program hosted at, and supported by, the Newton Institute Cambridge United Kingdom. NPS would like to acknowledge support from the United Kingdom Engineering and Physical Sciences Research Council (FP/F059361/1), Biotechnology and Biological Sciences Research Council (BB/F01080X/1) and the European Commission (FP7-ICT-2007-224495: euHeart). FBS acknowledges support by the Nora Eccles Treadwell Foundation. This publication is based on work (by EJC) supported in part by Award No KUK-C1-013-04, made by King Abdullah University of Science and Technology (KAUST). The authors also extend thanks to Liz Gresham for her proofreading of the manuscript.

\section{References}

Akaike, H., 1974. A new look at the statistical model identification. IEEE Trans. Automat. Contr. 19, 716-723.

Armstrong, C.M., Bezanilla, F., 1973. Currents related to movement of the gating particles of the sodium channels. Nature 242(5398), 459-61.

Aslanidi, O.V., Stewart, P., Boyett, M.R., Zhang, H., 2009. Optimal velocity and safety of discontinuous conduction through the heterogeneous Purkinje-ventricular junction. Biophys. J. 97, 20-39.

Banga, J.R., Balsa-Canto, E., 2008. Parameter estimation and optimal experimental design. Essays Biochem. 45, 195-209.

Bassani, R. A., Bassani, J. W., Bers, D. M., 1992. Mitochondrial and sarcolemmal Ca ${ }^{2+}$ transport reduce $\left[\mathrm{Ca}^{2+}\right]_{\mathrm{i}}$ during caffeine contractures in rabbit cardiac myocytes. J. Physiol. 453, 591-608.

Berman, H., Henrick, K., Nakamura, H., 2003. Announcing the worldwide Protein Data Bank. Nat. Struct. Biol. 10, 980.

Bernus, O., Wilders, R., Zemlin, C.W., Verschelde, H., Panfilov, A.V., 2002. A computationally efficient electrophysiological model of human ventricular cells. Am. J. Physiol. Heart Circ. Physiol. 282(6), H2296-2308.

Berridge, M., Lipp, P., Bootmann, M., 2000. The versatility and universality of calcium signaling. Nat. Rev. Mol. Cell Biol. 1(1), 11-21.

Bers, D., 2008. Calcium cycling and signaling in cardiac myocytes. Annu. Rev. Physiol. 70, 23-49.

Biktashev, V.N., Suckley, R., Elkin, Y.E., Simitev, R.D., 2008. Asymptotic analysis and analytical solutions of a model of cardiac excitation. Bull. Math. Biol. 70(2), 517554.

Biktasheva, I.V., Simitev, R.D., Suckley, R., Biktashev, V.N., 2006. Asymptotic properties of mathematical models of excitability. Philos. Transact. A Math. Phys. Eng. Sci. 364(1842), 1283-1298.

Bolte, S., Cordelières F.P., 2006. A guided tour into subcellular colocalization analysis in light microscopy. J. Microsc. 224(3), 213-32. 
Bondarenko, V.E., Szigeti, G.P., Bett, G.C.L., Kim, S.-J., Rasmusson, R.L., 2004. Computer model of action potential of mouse ventricular myocytes. Am. J. Physiol. Heart Circ. Physiol. 287, H1378-1403.

Boyett, M.R., Zhang, H., Garny, A., Holden, A.V., 2001. Control of the pacemaker activity of the sinoatrial node by intracellular $\mathrm{Ca}^{2+}$. Experiments and modelling. Phil. Trans. R. Soc. Lond. A 359, 1091-1110.

Boyett, M.R., Honjo, H., Kodama, I., 2000. The sinoatrial node, a heterogeneous pacemaker structure. Cardiovasc. Res. 47,658 -687.

Brazma, A., Hingamp, P., Quackenbush, J., Sherlock, G., Spellman, P., Stoeckert, C., Aach, J., Ansorge, W., Ball C.A., Causton, H.C., Gaasterland, T., Glenisson, P., Holstege, F.C., Kim, I.F., Markowitz, V., Matese, J.C., Parkinson, H., Robinson, A., Sarkans, U., Schulze-Kremer, S., Stewart, J., Taylor, R., Vilo, J., Vingron, M., 2001. Minimum information about a microarray experiment (MIAME) - toward standards for microarray data. Nat. Genet. 29, 365-371.

Brazma, A., Parkinson, H., Sarkans, U., Shojatalab, M., Vilo, J., Abeygunawardena, N., Holloway, E., Kapushesky, M., Kemmeren, P., Lara, G.G., Oezcimen, A., RoccaSerra, P., Sansone, S.A., 2003. ArrayExpress - a public repository for microarray gene expression data at the EBI. Nucleic Acids Res. 31, 68 -71.

Brette, R., Rudolph, M., Carnevale, T., Hines, M., Beeman, D., Bower, J.M., Diesmann, M., Goodman, P.H., Harris, F.C.J., Zirpe, M., Natschläger, T., Pecevski, D., Ermentrout, B., Djurfeldt, M., Lansner, A., Rochel, O., Vieville, T., Muller, E., Davison, A., El Boustani, S., Destexhe, A., 2007. Simulation of networks of spiking neurons: a review of tools and strategies. J. Comput. Neurosci. 23, 349-398.

Bueno-Orovio, A., Cherry, E.M., Fenton, F.H., 2008. Minimal model for human ventricular action potentials in tissue. J. Theor. Biol. 253(3), 544-560.

Burnham, K.P., Anderson, D.R., 2002. Model Selection and Multimodel Inference: A Practical-Theoretic Approach, second ed. Springer-Verlag. ISBN 0-387-95364-7.

Cabo, C., Boyden, P.A., 2003. Electrical remodeling of the epicardial border zone in the canine infarcted heart: a computational analysis. Am. J. Physiol. Heart Circ. Physiol. 284, H372-384.

Cain, J.W., Schaeffer, D.G., 2006. Two-term asymptotic approximation of a cardiac restitution curve. SIAM Rev. Soc. Ind. Appl. Math. 48(3), 537-546.

Campbell SG, Flaim SN, Leem CH, McCulloch AD., 2008. Mechanisms of transmurally varying myocyte electromechanics in an integrated computational model. Philos Transact A Math Phys Eng Sci. 366(1879):3361-80.

Campbell, S.G., Howard, E., Aguado-Sierra, J., Coppola, B.A., Omens, J.H., Mulligan, L.J., McCulloch, A.D., Kerckhoffs, R.C., 2009. Effect of transmurally heterogeneous myocyte excitation-contraction coupling on canine left ventricular electromechanics. Exp. Physiol. 94(5), 541-52

Carpenter, J., Bithell, J., 2000. Bootstrap confidence intervals: when, which, what? A practical guide for medical statisticians. Stat. Med. May 15, 19(9), 1141-64.

Chandler, N.J., Greener, I.D., Tellez, J.O., Inada, S., Musa, H., Molenaar, P., Difrancesco, D., Baruscotti, M., Longhi, R., Anderson, R.H., Billeter, R., Sharma, V., Sigg, D.C., Boyett, M.R., Dobrzynski, H., 2009. Molecular architecture of the human sinus node: insights into the function of the cardiac pacemaker. Circulation 19(12), 1562-75. 
Cheng, H., Lederer, W.J., Cannell, M.B.,1993. Calcium sparks: elementary events underlying excitation-contraction coupling in heart muscle. Science 262(5134), $740-4$.

Cherry, E.M., Evans, S.J., 2008. Properties of two human atrial cell models in tissue: restitution, memory, propagation, and reentry. J. Theor. Biol. 254, 674-90.

Cherry, E.M., Fenton, F.H., 2004. Suppression of alternans and conduction blocks despite steep APD restitution: electrotonic, memory, and conduction velocity restitution effects. Am. J. Physiol. Heart Circ. Physiol. 286, H2332-41.

Cherry, E.M., Fenton, F.H., 2007. A tale of two dogs: analyzing two models of canine ventricular electrophysiology. Am. J. Physiol. Heart Circ. Physiol. 292, H43-55.

Cherry, E.M., Hastings, H.M., Evans, S.J., 2008. Dynamics of human atrial cell models: restitution, memory, and intracellular calcium dynamics in single cells. Prog. Biophys. Mol. Biol. 98, 24-37.

Chopra, N., Kannankeril, P., Yang, T., Hlaing, T., Holinstat, I., Ettensohn, K., Pfeifer, K., Akin, B., Jones, L., Franzini-Armstrong, C., Knollmann, B., 2007. Modest reductions of cardiac calsequestrin increase sarcoplasmic reticulum $\mathrm{Ca}^{2+}$ leak independent of luminal $\mathrm{Ca}^{2+}$ and trigger ventricular arrhythmias in mice. Circ. Res.101(6), 617-26.

Chudin, E., Goldhaber, J., Garfinkel, A., Weiss, J., Kogan, B., 1999. Intracellular $\mathrm{Ca}^{2+}$ dynamics and the stability of ventricular tachycardia. Biophys. J. 77, 2930-2941.

Cooling, M., Hunter, P.J., Crampin, E.J., 2007. Modelling hypertrophic IP3 transients in the cardiac myocyte. Biophys. J. 93, 3421-3433.

Cooling, M., Hunter, P., Crampin, E.J., 2009. Sensitivity of NFAT cycling to cytosolic calcium concentration: implications for hypertrophic signals in cardiac myocytes. Biophys. J. 96(6), 2095-104.

Coombes, S., Hinch, R., Timofeeva, Y., 2004. Receptors, sparks and waves in a firediffuse-fire framework for calcium release. Prog. Biophys. Mol. Biol. 85, 197-219.

Coombes, S., Timofeeva, Y., 2003. Sparks and waves in a stochastic fire-diffuse-fire model of $\mathrm{Ca}^{2+}$ release. Phys. Rev. E: Stat., Nonlinear, Soft Matter Phys. 68, 021915-1-8.

Cornish-Bowden, A., 1977. An automatic method for deriving steady state rate equations. Biochem. J. 165 (1), 55-59.

Cortassa, S., Aon, M.A., Marbán, E., Winslow, R.L., O’Rourke, B., 2003. An integrated model of cardiac mitochondrial energy metabolism and calcium dynamics. Biophys. J. 84(4), 2734-2755.

Courtemanche, M., Ramirez, R.J., Nattel, S., 1998. Ionic mechanisms underlying human atrial action potential properties: insights from a mathematical model. Am. J. Physiol. 275, H301-321.

Crampin, E.J., Halstead, M., Hunter, P.J., Nielsen, P.F., Noble, D., Smith, N.P., Tawhai, M.H. 2004. Computational physiology and the Physiome project, Exp. Physiol. 89(1), 1-26.

Daniel, T.L., Trimble, A.C., Chase, P.B., 1998. Compliant realignment of binding sites in muscle: transient behavior and mechanical tuning. Biophys. J. 74, 1611-1621.

Dash, R.K., Qiand, F., Beard, D.A., 2009. A biophysically based mathematical model for the kinetics of mitochondrial calcium uniporter. Biophys. J. 96 (4), 1318-1332. 
Davis, J.P., Tikunova, S.B., 2008. $\mathrm{Ca}^{2+}$ exchange with troponin $\mathrm{C}$ and cardiac muscle dynamics. Cardiovasc. Res. 77, 619-626.

Dawson, S.P., Keizer, J., Pearson, J.E., 1999. Fire-diffuse-fire model of dynamics of intracellular calcium waves. Proc. Natl. Acad. Sci. U.S.A. 96(11), 6060-6063.

Dedkova, E.N., Blatter, L.A., 2008. Mitochondrial $\mathrm{Ca}^{2+}$ and the heart. Cell Calcium 44(1), 77-91.

DeFelice, L.D., 1997. Electrical Properties of the Cells. Patch Clamp for Biologists. Plenum Publishing Corporation.

deHass, V., Vogel, W., 1989. Sodium and potassium currents recorded during an action potential. Eur. Biophys. J. 17(1), 49-51.

Demir, S.S., Clark, J.W., Murphey, C.R., Giles, W.R., 1994. A mathematical model of a rabbit sinoatrial node cell. Am. J. Physiol. 266, C832-852.

DiFrancesco, D., Noble, D., 1985. A model of cardiac electrical activity incorporating ionic pumps and concentration changes. Philos. Trans. R. Soc. Lond. B, Biol. Sci. 307, 353-398.

Dobrunz, L.E., Backx, P.H., Yue, D.T., 1995. Steady-state $\left[\mathrm{Ca}^{2+}\right]$ i-force relationship in intact twitching cardiac muscle: direct evidence for modulation by isoproterenol and EMD 53998. Biophys. J. 69, 189-201.

Dokos, S., Celler, B., Lovell, N., 1996. Ion currents underlying sinoatrial node pacemaker activity: a new single cell mathematical model. J. Theor. Biol. 181, 245-272.

Dokos, S., Lovell, N.H., 2004. Parameter estimation in cardiac ionic models. Prog. Biophys. Mol. Biol. 85, 407-431.

Duchen, M.R., Leyssens, A., Crompton, M., 1998. Transient mitochondrial depolarizations reflect focal sarcoplasmic reticular calcium release in single rat cardiomyocytes. J. Cell Biol,142(4), 975-988.

Dupont, G., Goldbeter, A., 1994. Properties of intracellular $\mathrm{Ca}^{2+}$ waves generated by a model based on $\mathrm{Ca}^{2+}$-induced $\mathrm{Ca}^{2+}$ release. Biophys. J. 67(6), 2191-2204.

Edgar, R., Domrachev, M., Lash, A.E., 2002. Gene Expression Omnibus: NCBI gene expression and hybridization array data repository. Nucleic Acids Res. 30,207-210.

Eisenberg, E., Moos, C., 1968. Adenosinetriphosphatase activity of acto-heavy meromyosin. Kinetic analysis of actin activation. Biochemistry 7, 1486-1489.

Eisner, D.A., Choi, H.S., Díaz, M.E., O'Neill, S.C., Trafford, A.W., 2000. Integrative analysis of calcium cycling in cardiac muscle. Circ. Res. 87(12), 1087-1094.

Espinosa, L., 1998. L'Echange $\mathrm{Na}^{+} / \mathrm{Ca}^{2+}$ dans l'Hypertrophie Ventriculaire D'Altitude chez le Rat: Etude Electrophysiologique et Utilisation du Modele "Oxsoft Heart". $\mathrm{PhD}$ thesis, Leon.

Faber, G.M., Rudy, Y., 2000. Action potential and contractility changes in $\left[\mathrm{Na}^{+}\right](\mathrm{i})$ overloaded cardiac myocytes: a simulation study. Biophys. J., 78, 2392-2404.

Fenton, F., Karma, A., 1998. Vortex dynamics in three-dimensional continuous myocardium with fiber rotation: Filament instability and fibrillation. Chaos $8(1)$, $20-47$.

Fenton, F.H., Cherry, E.M., 2008. Models of cardiac cell. Scholarpedia 3, 1868.

Fink, M., Noble, D., 2009. Markov models for ion channels: versatility vs. identifiability and speed. Phil. Trans. R. Soc. Lond. A 367, 2161-2179.

Flaim, S.N., Giles, W.R., McCulloch, A.D., 2006. Contributions of sustained INa and IKv43 to transmural heterogeneity of early repolarization and arrhythmogenesis in 
canine left ventricular myocytes. Am. J. Physiol. Heart Circ. Physiol. 291, H26172629.

Fletcher, R., 1987. Practical Methods of Optimization. Wiley, New York.

Fluhler, E., Burnham, V.G., Loew, L.M, 1985. Spectra, membrane binding, and potentiometric responses of new charge shift probe. Biochemistry 24(21), 5749-55.

Foracchia, M., Hooker, A., Vicini, P., Ruggeri, A., 2004. POPED, a software for optimal experimental design in population kinetics. Comput. Methods Programs Biomed. 74, 29-46.

Fox, J.J., McHarg, J.L., Gilmour, R.F., 2002. Ionic mechanism of electrical alternans. Am. J. Physiol. Heart Circ. Physiol. 282(2), H516-H530.

Garny, A., Kohl, P., Noble, D., 2003. Cellular Open Resource (COR): A public CellML based environment for modeling biological function. Int. J. Bifurcat. Chaos 12, 3579-3590.

Garny, A., Nickerson, D.P., Cooper, J., Weber dos Santos, R., Miller, A.K., McKeever, S., Nielsen, P.M., Hunter, P.J., 2008. CellML and associated tools and techniques. Philos. Transact. A Math. Phys. Eng. Sci. 366(1878), 3017-43.

Gill, P.E., 1981. Practical Optimization. Academic Press, London.

Gin, E., Falcke, M., Wagner, L.E., Yule, D.I., Sneyd, J., 2009. Markov chain Monte Carlo fitting of single-channel data from inositol trisphosphate receptors, J. Theor. Biol. 257, 460-474.

Greenstein, J.L., Winslow, R.L., 2002. An integrative model of the cardiac ventricular myocyte incorporating local control of $\mathrm{Ca}^{2+}$ release. Biophys. J., 83, 2918-2945.

Guevara, M.R., Ward, G., Shrier, A., Glass, L., 1984. Electrical alternans and perioddoubling bifurcations. Comput. Cardiol. 167-170.

Gutenkunst, R.N., Waterfall, J.J., Casey, F.P., Brown, K.S., Myers, C.R., Sethna, J.P., 2007. Universally sloppy parameter sensitivities in systems biology models. PLoS Comput. Biol. Oct 3(10), 1871-78.

Györke, I., Hester, N., Jones, L.R., Györke, S., 2004. The role of Calsequestrin, Triadin, and Junctin in conferring cardiac ryanodine receptor responsiveness to luminal Calcium. Biophys. J. 86(4), 2121-2128.

Györke, S., Terentyev, D., 2008. Modulation of ryanodine receptor by luminal calcium and accessory proteins in health and cardiac disease. Cardiovasc. Res. 77(2), 245255.

Hilgemann, D.W., Noble, D., 1987. Excitation-contraction coupling and extracellular calcium transients in rabbit atrium: reconstruction of basic cellular mechanisms. Proc. R. Soc. Lond. B230, 163-205.

Hill, T.L., Eisenberg, E., Chalovich, J M., 1981. Theoretical models for cooperative steady-state ATPase activity of myosin subfragment-1 on regulated actin. Biophys. J. 35, 99-112.

Hille, B., 2001. Ion Channels of Excitable Membranes, third ed. Sinauer Associates, Sunderland, MA.

Hinch, R., 2004. A mathematical analysis of the generation and termination of calcium sparks. Biophys. J. 86(3), 1293-307.

Hinch, R., Greenstein, J.L., Tanskanen, A.J., Xu, L., Winslow, R.L., 2004. A simplified local control model of calcium-induced calcium release in cardiac ventricular myocytes. Biophys. J. 87, 3723-3736. 
Hodgkin, A.L., Huxley, A.F., 1952. A quantitative description of membrane current and its application to conduction and excitation in nerve. J. Physiol. 117, 500-544.

Holmes, J. W., 2004. Candidate mechanical stimuli for hypertrophy during volume overload. J. Appl. Physiol. 97, 1453-1460.

Hui, B.B.B., Dokos, S., Lovell, N.H., 2007. Parameter identifiability of cardiac ionic models using a novel CellML least squares optimization tool. Conference Proceedings: Annual International Conference of the IEEE Engineering in Medicine and Biology Society 2007, 5307-5310.

Hucka, M., Finney, A., Bornstein, B.J., Keating, S.M., Shapiro, B.E., Matthews, J., Kovitz, B.L., Schilstra, M.J., Funahashi, A., Doyle, J.C., Kitano, H., 2004. Evolving a lingua franca and associated software infrastructure for computational systems biology: the Systems Biology Markup Language (SBML) project. Syst. Biol. (Stevenage) 1, 41-53.

Hund, T.J., Rudy, Y., 2004. Rate dependence and regulation of action potential and calcium transient in a canine cardiac ventricular cell model. Circulation 110, 316874.

Hunter, P.J., 2004. The IUPS Physiome Project: a framework for computational physiology. Prog. Biophys. Mol. Biol. 85, 551-569.

Hunter, P.J., Borg, T.K., 2003. Integration from proteins to organs: the Physiome Project. Nat. Rev. Mol. Cell Biol. 4, 237-243.

Huxley, A.F., 1957. Muscle structure and theories of contraction. Prog. Biophys. Mol. Biol. 7, 255.

Huxley, A.F., Simmons, R.M., 1971. Proposed mechanism of force generation in striated muscle. Nature 233, 533.

Ikeo, K., Ishi-i, J., Tamura, T., Gojobori, T., Tateno, Y., 2003. CIBEX: center for information biology gene expression database. C. R. Biol. 326, 1079-1082.

Iribe, G., Helmes, M., Kohl, P., 2007. Force-length relations in isolated intact cardiomyocytes subjected to dynamic changes in mechanical load. Am. J. Physiol. Heart Circ. Physiol. 292, H1487-1497.

Iyer, V., Mazhari, R., Winslow, R.L., 2004. A computational model of the human leftventricular epicardial myocyte. Biophys. J., 87, 1507-1525.

Izu, L.T., Means, S.A., Shadid, J.N., Chen-Izu, Y., Balke, C.W., 2006. Interplay of ryanodine receptor distribution and calcium dynamics. Biophys. J. 91(1), 95-112.

Izu, L.T., Wier, W.G., Balke, C.W., 2001. Evolution of cardiac calcium waves from stochastic calcium sparks. Biophys. J. 80(1), 103-120.

Kahlig, K.M., Misra, S.N., George, A.L., Jr, 2006. Impaired inactivation gate stabilization predicts increased persistent current for an epilepsy-associated SCN1A mutation. J. Neurosci. 26(43), 10958-10966.

Karma, A., 1993. Spiral breakup in model equations of action potential propagation in cardiac tissue. Phys. Rev. Lett. 71, 1103-1106.

Keener, J. P., 2009. Invariant manifold reductions for Markovian ion channel dynamics. J. Math. Biol. 58(3), 447-57.

Keizer, J., Smith, G.D., Ponce-Dawson, S., Pearson, J.E., 1998. Saltatory propagation of $\mathrm{Ca}^{2+}$ waves by $\mathrm{Ca}^{2+}$ sparks. Biophys. J. 75(2), 595-600.

Kennedy, J., Eberhart, R., 1995. Particle swarm optimization, in: Proc. IEEE Int. Conf. on Neural Networks, Piscataway, NJ, pp. 1942-1948. 
Kerckhoffs, R.C.P., Bovendeered, P.H.M., Kotte, C.C.S., Prinzen, F.W., Smits, K., Arts, T., 2003. Homogenity of cardiac contraction despite physiological asynchrony of depolarization: A model study. Ann. Biomed. Eng. 51(5) 536-547.

Kharche, S., Lüdtke, N., Panzeri, S., Zhang, H., 2009. A global sensitivity index for biophysically detailed cardiac cell models: a computational approach. LNCS 5528, 366-375.

Kirkpatrick, S., Gelatt, C.D., Vecchi, M.P., 1983. Optimization by simulated annealing. Science New Series 220(4598), 671-680.

Korhonen, T., Hanninen, S., Tavi, P., 2009. Model of excitation-contraction coupling of rat neonatal ventricular myocytes. Biophys. J. 96(3), 1189-1209.

Kurata, Y., Hisatome, I., Imanishi, S., Shibamoto, T., 2002. Dynamical description of sinoatrial node pacemaking: improved mathematical model for primary pacemaker cell. Am. J. Physiol. Heart Circ. Physiol. 283, H2074-2101.

Kurata, Y., Matsuda, H., Hisatome, I., Shibamoto, T., 2008. Regional difference in dynamical property of sinoatrial node pacemaking: role of $\mathrm{Na}^{+}$channel current. Biophys. J. 95, 951-977.

Langer, G.A., Peskoff, A., 1996. Calcium concentration and movement in the diadic cleft space of the cardiac ventricular cell. Biophys. J. 70(3), 1169-1182.

Lasher, R.A., Hitchcock, R.W., Sachse, F.B., 2009. Towards modeling of cardiac microstructure with catheter-based confocal microscopy: a novel approach for dye delivery and tissue characterization. IEEE Trans. Med. Imaging 28(8), 1156-64.

Lemon, G., 2004. Fire-diffuse-fire calcium waves in confined intracellular spaces. Bull. Math. Biol. 66, 65-90.

Li, P., Holden, A., 2009. Intracellular $\mathrm{Ca}^{2+}$ nonlinear wave behaviours in a three dimensional ventricular cell model. Physica D 238 (11-12), 992-999.

Li, P., Lancaster, M., Holden, A., 2007. A three dimensional ventricular e-cell (3dv ecell) with stochastic intracellular $\mathrm{Ca}^{2+}$ handling, in: Functional Imaging and Modeling of the Heart. Lecture Notes in Computer Science. Springer Berlin/Heidelberg, pp. 180-189.

Lindblad, D.S., Murphey, C.R., Clark, J.W., Giles, W.R., 1996. A model of the action potential and underlying membrane currents in a rabbit atrial cell. Am. J. Physiol. 271, H1666-1696.

Livshitz, L. M., Rudy, Y., 2007. Regulation of $\mathrm{Ca}^{2+}$ and electrical alternans in cardiac myocytes: role of CAMKII and repolarizing currents. Am. J. Physiol. 292, H28542866.

Lu S, Michailova A, Saucerman J, Cheng Y, Yu Z, Kaiser T, Li W, Bank R, Holst M, McCammon J, Hayashi T, Hoshijima M, Arzberger P, McCulloch A.,2009, Multiscale modeling in rodent ventricular myocytes. IEEE Eng Med Biol Mag. 28(2):46-57.

Luo, C.H., Rudy, Y., 1991. A model of the ventricular cardiac action potential. Depolarization, repolarization, and their interaction. Circ. Res. 68, 1501-1526.

Luo, C.H., Rudy, Y., 1994. A dynamic model of the cardiac ventricular action potential. I. Simulations of ionic currents and concentration changes. Circ. Res. 74, 1071-96.

Lymn, R.W., Taylor, E.W., 1971. Mechanism of adenosine triphosphate hydrolysis by actomyosin. Biochemistry 10, 4617.

Mahajan, A., Shiferaw, Y., Sato, D., Baher, A., Olcese, R., Xie, L.-H., Yang,M.-J., Chen, 
P.-S., Restrepo, J. G., Karma, A., Garfinkel, A., Qu, Z., Weiss, J.N., 2008. A rabbit ventricular action potential model replicating cardiac dynamics at rapid heart rates. Biophys. J. 94(2), 392-410.

Matiukas, A., Mitrea, B.G., Pertsov, A.M., Wuskell, J.P., Wei, M.D., Watras, J., Millard, A.C., Loew, L.M., 2006. New near-infrared optical probes of cardiac electrical activity. Am. J. Physiol. Heart Circ. Physiol. 290(6), H2633-43.

Matsuoka, S., Sarai, N., Kuratomi, S., Ono, K., Noma, A., 2003. Role of individual ionic current systems in ventricular cells hypothesized by a model study. Jpn. J. Physiol. $53,105-123$.

McAllister, R.E., Noble, D., Tsien, R.W., 1975. Reconstruction of the electrical activity of cardiac Purkinje fibres. J. Physiol. 251, 1-59.

Means, S., Smith, A.J., Shepherd, J., Shadid, J., Fowler, J., Wojcikiewicz, R.J.H., Mazel, T., Smith, G.D., Wilson, B.S., 2006. Reaction diffusion modeling of calcium dynamics with realistic ER geometry. Biophys. J. 91(2), 537-557.

Meza, C., Jaffrezic, F., Foulley, J.L., 2007. REML estimation of variance parameters in non linear mixed effects models using the SAEM algorithm. Biom. J. 49, 1-13.

Michailova, A., DelPrincipe, F., Egger, M., Niggli, E., 2002. Spatiotemporal features of $\mathrm{Ca}^{2+}$ buffering and diffusion in atrial cardiac myocytes with inhibited sarcoplasmic reticulum. Biophys. J. 83, 3134-3151.

Milescu, L.S., Akk, G., Sachs, F., 2005. Maximum likelihood estimation of ion channel kinetics from macroscopic currents. Biophys. J. 88(4), $2494-2515$.

Neher, E., Sakmann, B., 2007. Single-Channel Recording. Springer. ISBN-10: 030644870X.

Nguyen, M.-H.T., Dudycha, S.J., Jafri, M.S., 2007. Effect of $\mathrm{Ca}^{2+}$ on cardiac mitochondrial energy production is modulated by $\mathrm{Na}^{+}$and $\mathrm{H}^{+}$dynamics. Am. J. Physiol. Cell Physiol.292, C2004-C2020.

Nguyen, V., Mathias, R., Smith, G.D., 2005. A stochastic automata network descriptor for Markov chain models of instantaneously coupled intracellular $\mathrm{Ca}^{2+}$ channels. Bull. Math. Biol. 67, 393-432.

Niederer, S.A., Fink, M., Noble, D., Smith, N.P., 2009. A meta-analysis of cardiac electrophysiology computational models. Exp. Physiol. 94(5), 486-495.

Niederer, S.A., Hunter, P.J., Smith, N.P., 2006. A quantitative analysis of cardiac myocyte relaxation: A simulation study. Biophys. J. 90, 1697-1722.

Niederer, S.A., Smith, N.P., 2007. A mathematical model of the slow force response to stretch in rat ventricular myocytes. Biophys. J. 92, 4030-44.

Niederer, S.A., Smith N.P., 2009. The role of the Frank-Starling Law in the transduction of cellular work to whole organ pump function: a computational modeling analysis. PLoS Comput. Biol. 5(4), e1000371.

Nielsen, P., Hunter, P.J., 2008. CellML and associated tools and techniques. Philosophical Transactions of the Royal Society A.

Nilius, B., Hess, P., Lansman, J.B., Tsien, R.W., 1985. A novel type of cardiac calcium channel in ventricular cells. Nature 316(6027), 443-6.

Noble, D., Noble, S.J., 1984. A model of sino-atrial node electrical activity based on a modification of the DiFrancesco-Noble (1984) equations. Proc. R. Soc. Lond. B Biol. Sci. 222, 295-304. 
Noble, D., 1962. A modification of the Hodgkin-Huxley equations applicable to Purkinje fibre action and pace-maker potentials. J. Physiol. 160, 317-352.

Noble, D., Noble, S.J., Bett, G.C., Earm, Y.E., Ho, W.K., So, I.K., 1991. The role of sodium-calcium exchange during the cardiac action potential. Ann. N. Y. Acad. Sci. 639, 334-353.

Noble, D., Varghese, A., Kohl, P., Noble, P., 1998. Improved guinea-pig ventricular cell model incorporating a diadic space, I-Kr and I-Ks, and length- and tensiondependent processes. Can. J. Cardiol.14, 123-134.

Nolasco, J.B., Dahlen, R.W., 1968. A graphic method for the study of alternation in cardiac action potentials. J. Appl. Physiol. 25, 191-196.

Nordin, C., 1993. Computer model of membrane current and intracellular $\mathrm{Ca}^{2+}$ flux in the isolated guinea pig ventricular myocyte. Am. J. Physiol. 265, H2117-2136.

Nygren, A., Fiset, C., Firek, L., Clark, J.W., Lindblad, D.S., Clark, R.B., Giles, W.R., 1998. Mathematical model of an adult human atrial cell: the role of $\mathrm{K}^{+}$currents in repolarization. Circ. Res. 82, 63-81.

Nygren, A., Leon, L.J., Giles, W.R., 2001. Simulations of the human atrial action potential. Philos. Transact. A Math. Phys. Eng. Sci. 359, 1111-1125.

Olveczky, B.P., Verkman, A.S., 1998. Monte Carlo analysis of obstructed diffusion in three dimensions: Application to molecular diffusion in organelles. Biophys. J. 74 (5), 2722-2730.

Pandit, S.V., Clark, R.B., Giles, W.R., Demir, S.S., 2001. A mathematical model of action potential heterogeneity in adult rat left ventricular myocytes. Biophys. J. 81, 3029-3051.

Pásek M, Brette F, Nelson A, Pearce C, Qaiser A, Christe G, Orchard CH., 2008a., Quantification of t-tubule area and protein distribution in rat cardiac ventricular myocytes. Prog Biophys Mol Biol. 96(1-3):244-257.

Pásek M, Simurda J, Orchard CH, Christé G., 2008b. A model of the guinea-pig ventricular cardiac myocyte incorporating a transverse-axial tubular system. Prog Biophys Mol Biol. 96(1-3):258-80.

Pearson, J.E., Ponce-Dawson, S., 1998. Crisis on skid row. Physica A 257 (1-4), 141148.

Priebe, L., Beuckelmann, D.J., 1998. Simulation study of cellular electric properties in heart failure. Circ. Res. 82, 1206-1223.

Puglisi, J.L., Bers, D.M., 2001. LabHEART: an interactive computer model of rabbit ventricular myocyte ion channels and Ca transport. Am. J. Physiol. Cell Physiol. 281, C2049-2060.

Qi, F., Dash, R.K., Han, Y., Beard, D.A., 2009. Generating rate equations for complex enzyme systems by a computer-assisted systematic method. BMC Bioinformatics $10,238$.

Qu, Z., Shiferaw, Y., Weiss, J.N., 2007. Nonlinear dynamics of cardiac excitationcontraction coupling: an iterated map study. Phys. Rev. E: Stat., Nonlinear, Soft Matter Phys. 75, 011927.

Ramirez, R.J., Nattel, S., Courtemanche, M., 2000. Mathematical analysis of canine atrial action potentials: rate, regional factors, and electrical remodeling. Am. J. Physiol. Heart Circ. Physiol. 279, H1767-1785. 
Razumova, M.V., Bukatina, A.E., Campbell, K.B., 1999. Stiffness-distortion sarcomere model for muscle simulation. J. Appl. Physiol. 87, 1861-1876.

Rice, J.J., de Tombe, P.P., 2004. Approaches to modeling crossbridges and calciumdependent activation in cardiac muscle. Prog. Biophys. Mol. Biol. 85, 179-195.

Rice, J.J., Stolovitzky, G., Tu, Y., de Tombe, P.P., 2003. Ising model of cardiac thin filament activation with nearest-neighbor cooperative interactions. Biophys. J. 84, 897-909.

Rice, J.J., Wang, F., Bers, D.M., de Tombe, P.P., 2008. Approximate model of cooperative activation and crossbridge cycling in cardiac muscle using ordinary differential equations. Biophys. J. 95, 2368-2390.

Rice, J.J., Winslow, R.L., Hunter, W.C., 1999. Comparison of putative cooperative mechanisms in cardiac muscle: length dependence and dynamic responses. Am. J. Physiol. 276, H1734-H1754.

Rissanen J., 1978. Modeling by shortest data description. Automatica 14, 465-471.

Rüdiger, S., Shuai, J. W., Huisinga, W., Nagaiah, C., Warnecke, G., Parker, I., Falcke, M., 2007. Hybrid stochastic and deterministic simulations of calcium blips. Biophys. J. 93(6), 1847-1857.

Rudy Y., 2000. From genome to physiome: integrative models of cardiac excitation. Ann. Biomed. Eng. 28, 945-950.

Sachse, F.B., Glänzel, K.G., Seemann, G., 2003. Modeling of protein interactions involved in cardiac tension development. Int. J. Bifurc. Chaos, 13(12), 3561-3578.

Sakmann, B.F., Spindler, A.J., Bryant, S.M., Linz, K.W., Noble, D., 2000. Distribution of a persistent sodium current across the ventricular wall in guinea pigs. Circ. Res. 87(10), 910-914.

Sarai, N., Matsuoka, S., Kuratomi, S., Ono, K., Noma, A., 2003. Role of individual ionic current systems in the SA node hypothesized by a model study. Jpn. J. Physiol. 53, $125-134$.

Saucerman, J.J., Brunton, L.L., Michailova, A.P., McCulloch, A.D., 2003. Modeling beta-adrenergic control of cardiac myocyte contractility in silico. J. Biol. Chem. 278(48), 47997-48003.

Schaeffer, D.G., Cain, J.W., Gauthier, D.J., Kalb, S.S., Oliver, R.A., Tolkacheva, E.G., Ying, W., Krassowska, W., 2007. An ionically based mapping model with memory for cardiac restitution. Bull. Math. Biol. 69, 459-482.

Schaeffer, D.G., Ying, W., Zhao, X., 2008. Asymptotic approximation of an ionic model for cardiac restitution. Nonlinear Dyn. 51, 189-198.

Schmidt, H., Madsen, M.F., Dano, S., Cedersund, G., 2008. Complexity reduction of biochemical rate expressions. Bioinformatics 24(6), 848-854.

Schneider, N.S., Shimayoshi, T., Amano, A., Matsuda, T., 2006. Mechanism of the Frank-Starling law-A simulation study with a novel cardiac muscle contraction model that includes titin and troponin I. J. Mol. Cell. Cardiol. 41, 522-536.

Seber, G.A.F., Wild, C.J., 2003. Nonlinear Regression. Wiley-Interscience, Hoboken, NJ.

Shannon, T.R., Ginsburg, K.S., Bers, D.M., 2000. Reverse mode of the sarcoplasmic reticulum calcium pump and load-dependent cytosolic calcium decline in voltageclamped cardiac ventricular myocytes. Biophys. J. 78, 322-333.

Shannon, T.R., Guo, T., Bers, D.M., 2003. $\mathrm{Ca}^{2+}$ scraps: local depletions of free $\left[\mathrm{Ca}^{2+}\right]$ in cardiac sarcoplasmic reticulum during contractions leave substantial $\mathrm{Ca}^{2+}$ reserve. 
Circ. Res. 93(1), 40-45.

Shannon, T.R., Wang, F., Puglisi, J., Weber, C., Bers, D.M., 2004. A mathematical treatment of integrated Ca dynamics within the ventricular myocyte. Biophys. J., 87, 3351-3371.

Smith, D.A., Geeves, M.A., Sleep, J., Mijailovich, S.M., 2008. Towards a unified theory of muscle contraction. I: Foundations. Ann. Biomed. Eng. 36(10), 1624-1640.

Smith, N.P., Crampin, E.J., 2004. Development of models of active ion transport for whole-cell modelling: Cardiac sodium-potassium pump as a case study. Prog. Biophys. Mol. Biol. 85(2-3), 387-405.

Smith, N.P., Barclay, C.J., Loiselle, D.S., 2005. The efficiency of muscle contraction. Prog. Biophys. Mol. Biol. 88(1) 1-58.

Smith, N.P., Crampin, E.J., Niederer, S.A., Bassingthwaighte, J.B., Beard, D.A., 2007. Computational biology of cardiac myocytes: proposed standards for the physiome. J. Exp. Biol. 210(9), 1576-83.

Sobie, E.A., 2009. Parameter sensitivity analysis in electrophysiological models using multivariable regression. Biophys. J., 96, 1264-1274.

Soeller, C., Jayasinghe, I.D., Li, P., Holden, A.V., Cannell, M.B., 2009. Threedimensional high-resolution imaging of cardiac proteins to construct models of intracellular $\mathrm{Ca}^{2+}$ signalling in rat ventricular myocytes. Exp. Physiol. 94(5), 496508.

Stein, L.A., Schwarz, R.P., Jr., Chock, P.B., Eisenberg, E., 1979. Mechanism of actomyosin adenosine triphosphatase. Evidence that adenosine 5'-triphosphate hydrolysis can occur without dissociation of the actomyosin complex. Biochemistry 18, 3895-909.

Stelzer JE, Norman HS, Chen PP, Patel JR, Moss RL.,2008. Transmural variation in myosin heavy chain isoform expression modulates the timing of myocardial force generation in porcine left ventricle. J Physiol. 586(21):5203-14.

Stewart, P., Aslanidi, O.V., Noble, D., Noble, P.J., Boyett, M.R., Zhang, H., 2009. Mathematical models of the electrical action potential of Purkinje fibre cells. Philos. Transact. A Math. Phys. Eng. Sci. 367, 2225-2255.

Swietach, P., Spitzer, K., Vaughan-Jones, R., 2008. $\mathrm{Ca}^{2+}$-mobility in the sarcoplasmic reticulum of ventricular myocytes is low. Biophys. J. 95, 1412-27.

Syed, Z., Vigmond, E., Leon, L., 2005. Suitability of genetic algorithm generated models to simulate atrial fibrillation and $\mathrm{K}^{+}$channel blockades. Conference Proceedings: Annual International Conference of the IEEE Engineering in Medicine and Biology Society 7, 7087-7090.

Taylor, C.F., Paton, N.W., Lilley, K.S., Binz, P.A., Julian, R.K., Jr, Jones, A.R., Zhu, W., Apweiler, R., Aebersold, R., Deutsch, E.W., Dunn, M.J., Heck, A.J., Leitner, A., Macht, M., Mann, M., Martens, L., Neubert, T.A., Patterson, S.D., Ping, P., Seymour, S.L., Souda, P., Tsugita, A., Vandekerckhove, J., Vondriska, T.M., Whitelegge, J.P., Wilkins, M.R., Xenarios, I., Yates, J.R., 3rd, Hermjakob, H., 2007. The minimum information about a proteomics experiment (MIAPE). Nat. Biotechnol. 25, 887-893.

ten Tusscher, K.H.W.J., Bernus, O., Hren, R., Panfilov, A.V., 2006. Comparison of electrophysiological models for human ventricular cells and tissues. Prog. Biophys. Mol. Biol. 90, 326-345. 
ten Tusscher, K.H.W.J., Noble, D., Noble, P.J., Panfilov, A.V., 2004. A model for human ventricular tissue. Am. J. Physiol. Heart Circ. Physiol. 286(4), H1573-89.

ten Tusscher, K.H.W.J., Panfilov, A.V., 2006. Alternans and spiral breakup in a human ventricular tissue model. Am. J. Physiol. Heart Circ. Physiol. 291, H1088-H1 100.

Thul, R., Smith, G., Coombes, S., 2008. A bidomain threshold model of propagating calcium waves. J. Math. Biol. 56(4), 435-463.

Thul, R., Thurley, K., Falcke, M., 2009. Towards a predictive model of $\mathrm{Ca}^{2+}$ puffs. Chaos in press.

Trafford, A., Diaz, M., Eisner, D., 1999. A novel, rapid and reversible method to measure $\mathrm{Ca}$ buffering and time-course of total sarcoplasmic reticulum $\mathrm{Ca}$ content in cardiac ventricular myocytes. Pflugers Arch. 437(3), 501-503.

Tran, K., Smith, N.P., Loiselle, D.S., Crampin, E.J., 2009. A thermodynamic model of the cardiac sarcoplasmic/endoplasmic $\mathrm{Ca}^{2+}$ (SERCA) pump. Biophys. J. 96, 2029204217.

Terkildsen, J., Niederer, S.A., Hunter, P.J., Crampin, E.J., Smith, N.P., 2008. Using Physiome Standards to couple cellular functions for cardiac excitation-contraction. Exp. Physiol. 93(7),919-29.

Trollinger, D.R., Cascio, W.E., Lemasters, J.J., 2000. Mitochondrial calcium transients in adult rabbit cardiac myocytes: inhibition by ruthenium red and artifacts caused by lysosomal loading of $\mathrm{Ca}^{2+}$-indicating fluorophores. Biophys. J. 79(1), 39-50.

Vanlier, J., Wu, F., Qi, F., Vinnakota, K.C., Han, Y., Dash, R.K., Yang, F., Beard, D.A., 2009. BISEN: Biochemical simulation environment. Bioinformatics 25(6) 836-837.

Varian, K.D., Raman, S., Janssen, P.M.L., 2006. Measurement of myofilament calcium sensitivity at physiological temperature in intact cardiac trabeculae. Am. J. Physiol. Heart Circ. Physiol. 290, H2092-2097.

Viceconti, M., Clapworthy, G., Van Sint Jan, S., 2008. The Virtual Physiological Human-A European initiative for in silico human modelling. J. Physiol. Sci. 7, 441-446.

Walden, A., Dibb, K., Trafford, A., 2009. Differences in intracellular calcium homeostasis between atrial and ventricular myocytes. J. Mol. Cell. Cardiol. 46(4), 463-73.

Wang, L.J., Sobie, E.A., 2008. Mathematical model of the neonatal mouse ventricular action potential. Am. J. Physiol. Heart. Circ. Physiol. 294, H2565-2575.

Weber, C.R., Piacentino, V., III, Ginsburg, K.S., Houser, S.R., Bers, D.M., 2002. Na ${ }^{+}$ $\mathrm{Ca}^{2+}$ exchange current and submembrane $\left[\mathrm{Ca}^{2+}\right]$ during the cardiac action potential. Circ. Res. 90(2), 182-189.

Wier, W.G., Cannel, M.B., Berlin, J.R., Marban, E., Lederer, W.J., 1987. Cellular and subcellular heterogeneity of $\mathrm{Ca}^{2+}$ in single heart cells revealed by fura-2. Science 235(4786), 325-328.

Wilders, R., Jongsma, H.J., van Ginneken, A.C., 1991. Pacemaker activity of the rabbit sinoatrial node. A comparison of mathematical models. Biophys. J. 60, 1202-1216.

Wilders, R., 2007. Computer modelling of the sinoatrial node. Med. Biol. Eng. Comput. 45, 189-207.

Winslow, R.L., Rice, J., Jafri, S., Marbán, E., O'Rourke, B., 1999. Mechanisms of altered excitation-contraction coupling in canine tachycardia-induced heart failure, II: model studies. Circ. Res. 84, 571-586. 
Wu, X., Bers, D., 2006. Sarcoplasmic reticulum and nuclear envelope are one highly interconnected $\mathrm{Ca}^{2+}$ store throughout cardiac myocyte. Circ. Res. 99(3), 283-91.

Wussling, M., Szymanski, G., 1986. Simulation by two calcium store models of myocardial dynamic properties: potentiation, staircase, and biphasic tension development. Gen. Physiol. Biophys. 5(2), 135-152.

Zaniboni, M., Rossini, A., Swietach, P., Banger, N., Spitzer, K.W., Vaughan-Jones, R.D., 2003. Proton permeation through the myocardial gap junction. Circ. Res. 93(8), 726-35.

Zhang, H., Holden, A.V., Kodama, I., Honjo, H., Lei, M., Varghese, T., Boyett, M.R., 2000. Mathematical models of action potentials in the periphery and center of the rabbit sinoatrial node. Am. J. Physiol. Heart Circ. Physiol. 279, H397-421.

Zou, G., Phillips, G.N., Jr., 1994. A cellular automaton model for the regulatory behavior of muscle thin filaments. Biophys. J. 67, 11-28. 

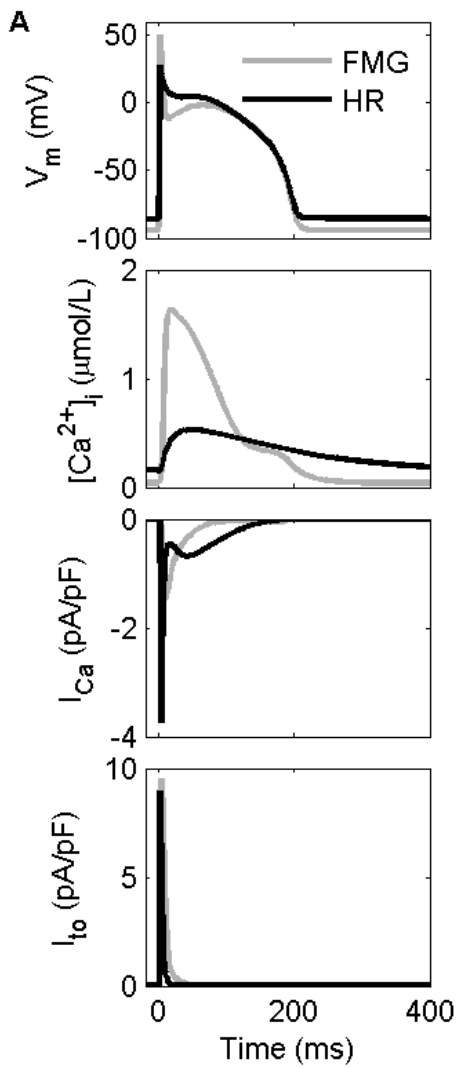

Figure 1. Comparison of action potentials, calcium transients, and selected ion currents in the Fox et al. (FMG) and Hund-Rudy (HR) canine ventricular models at pacing rates of (A) $500 \mathrm{~ms}$ and (B) $180 \mathrm{~ms}$.
B
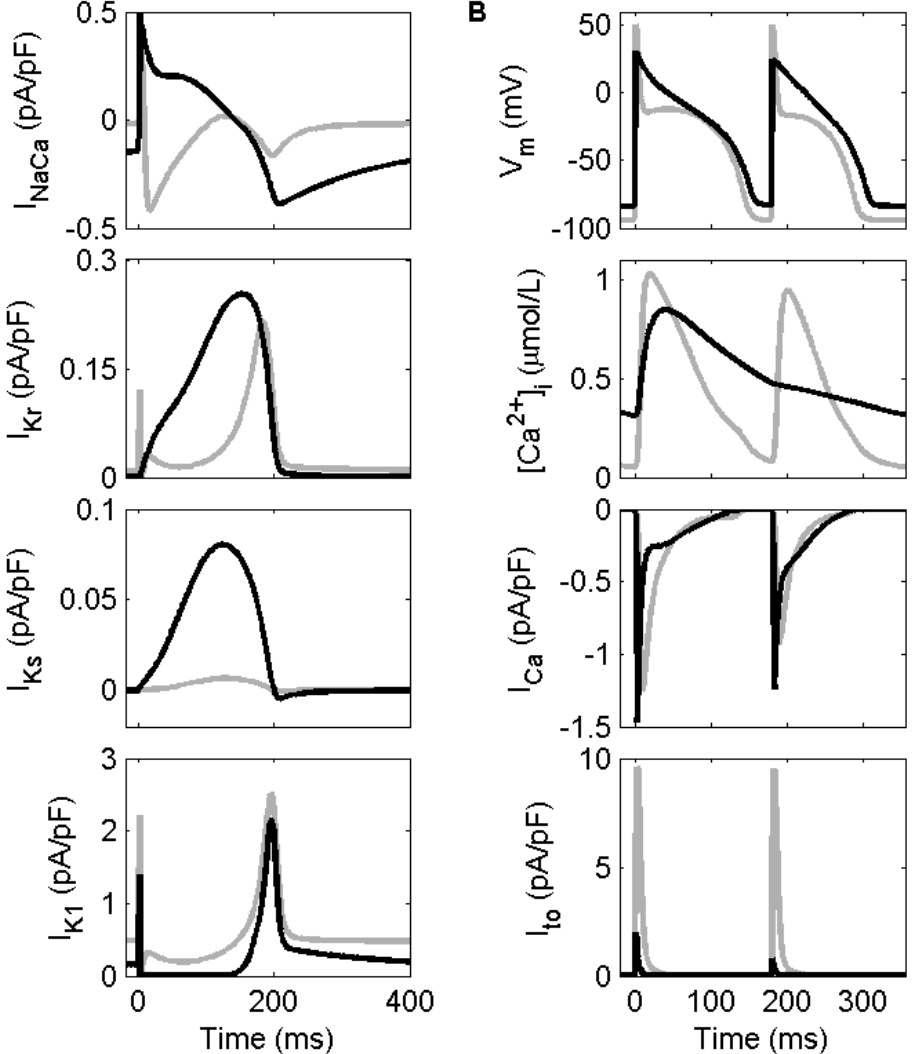


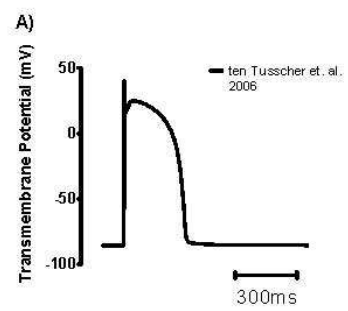

D)

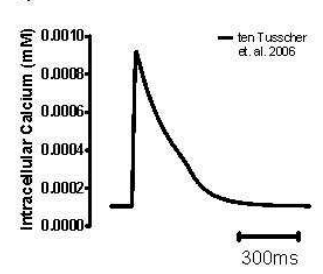

G)

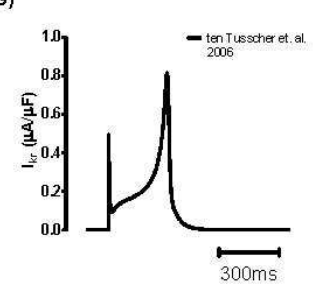

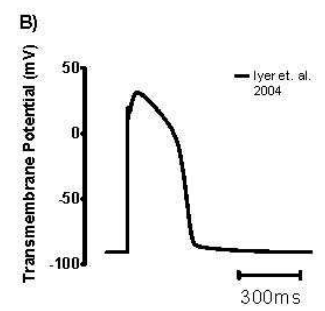

E)

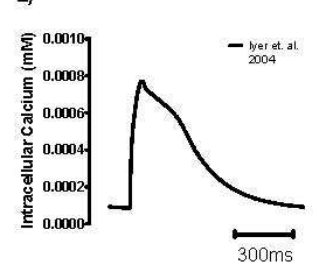

H)

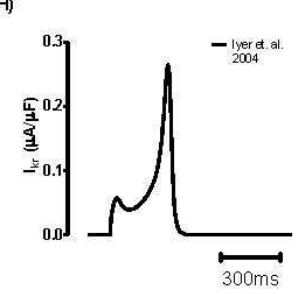

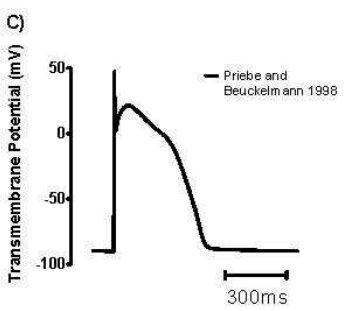

F)
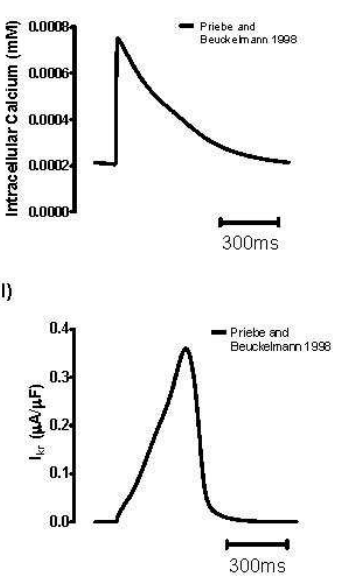

Figure 2

Differences in model predictions for the membrane potential, $\mathrm{Ca}^{2+}$ transient and $\mathrm{I}_{\mathrm{Kr}}$ channel current for three models of the human ventricular myocyte: A), D), G) ten Tusscher and Panfilov (2006) model; B), E), H) Iyer et al. (2004) model; C), F), I) Priebe and Beuckelmann (1998) model. 

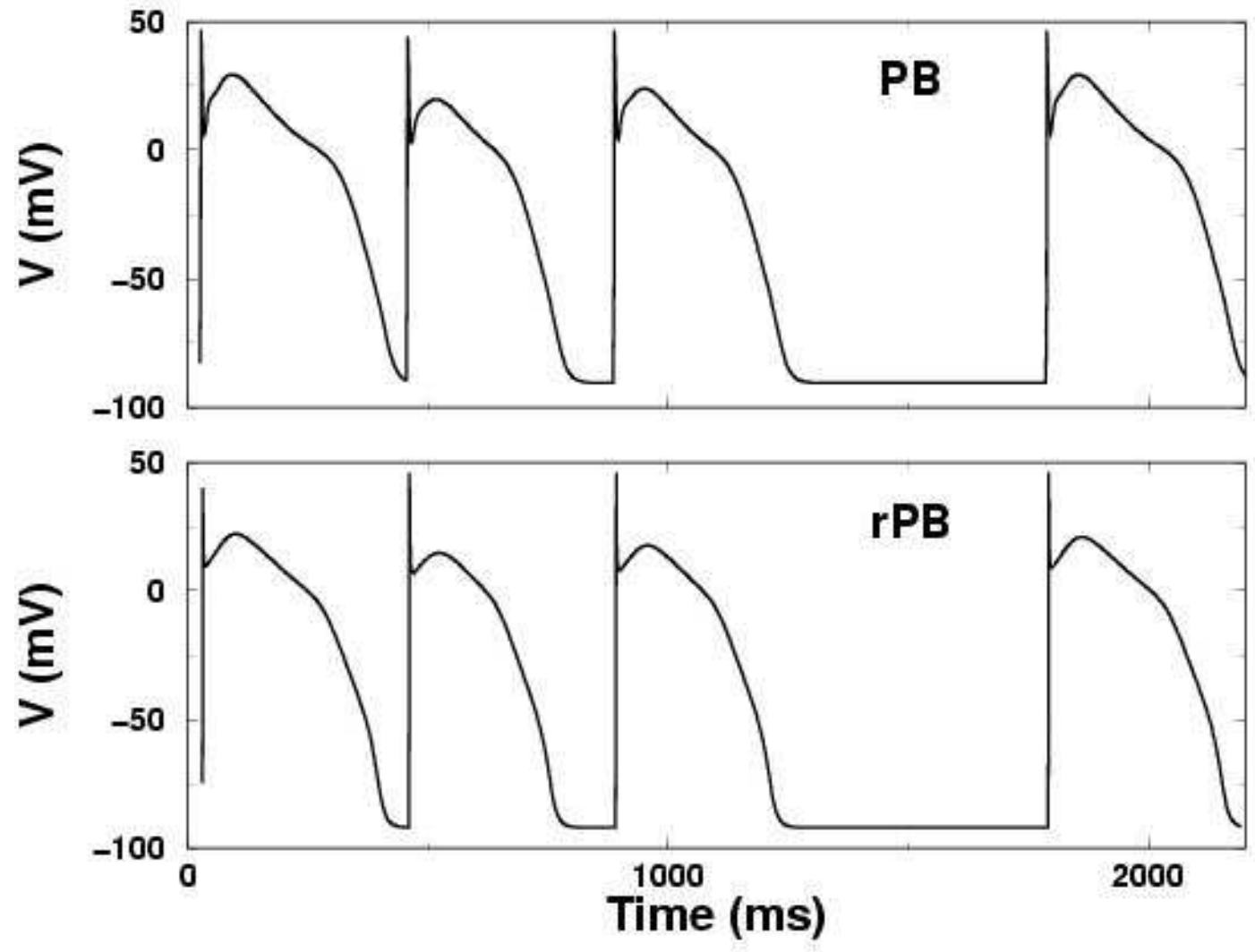

Figure 3. Four action potentials for the Priebe-Beuckelmann (PB) model and for the reduced PB (rPB) model of Bernus et al. (2002). Cycle length is $430 \mathrm{~ms}$ for the first two beats and $900 \mathrm{~ms}$ for the last two beats. 

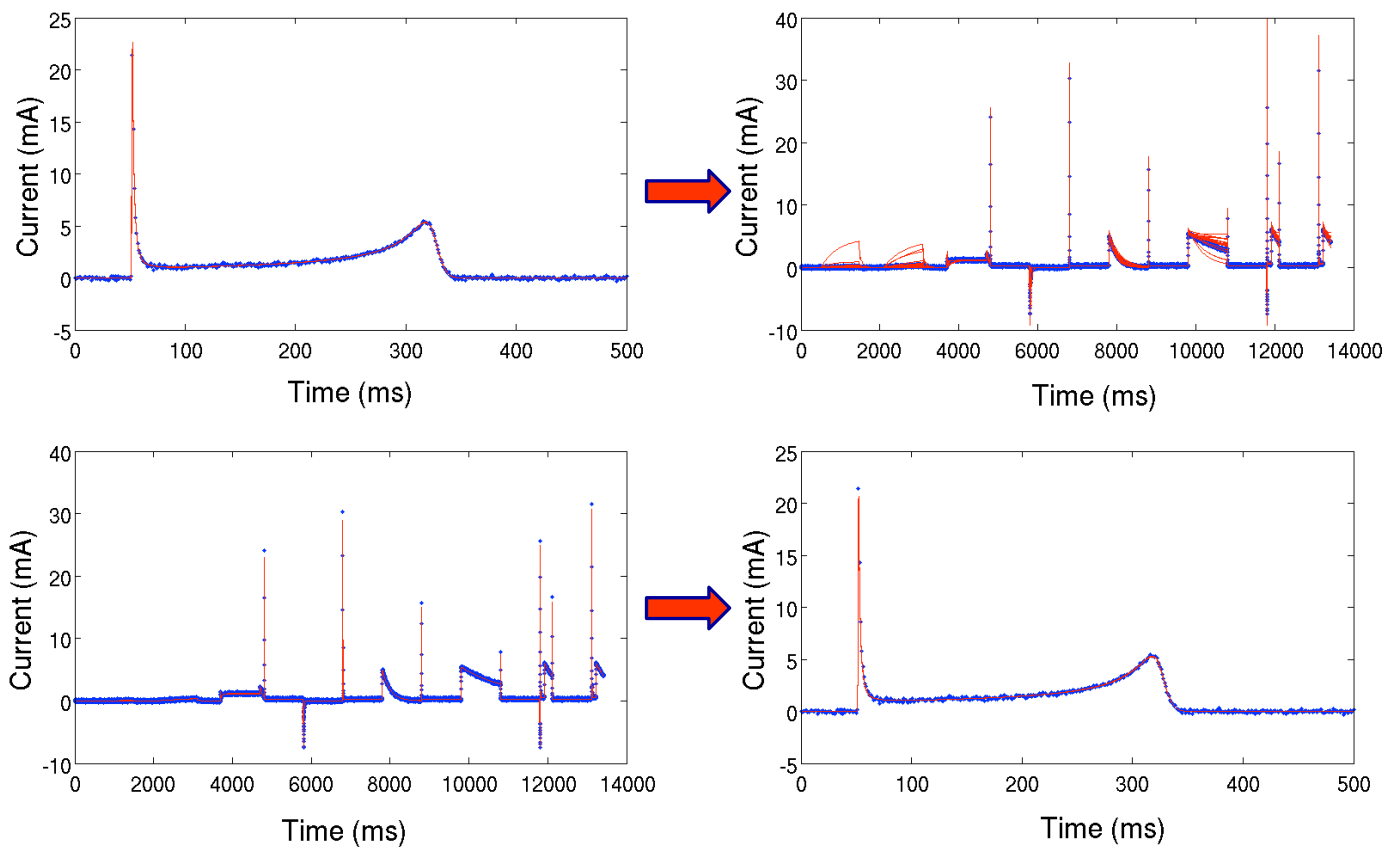

Figure 4: Fitting and validation of an $\mathrm{I}_{\mathrm{Kr}}$ model to AP-clamp data (top row) and data from an optimized voltage clamp (see Fink and Noble 2009) in the bottom row. A series of 20 fits of the $\mathrm{I}_{\mathrm{Kr}}$ model from the human ventricular cell model by ten Tusscher and Panfilov (2006) has been done in each case, where the red lines represent the fit and the blue dots the simulated data. All fits match the data almost perfectly, but when validating the fit by simulating the current during the optimised voltage clamp step protocol the fits show very different results, indicating that the information content in an action potential clamp is less than in the optimised protocol. The 20 fits to the optimised protocol match the data from the action potential clamp very well. 

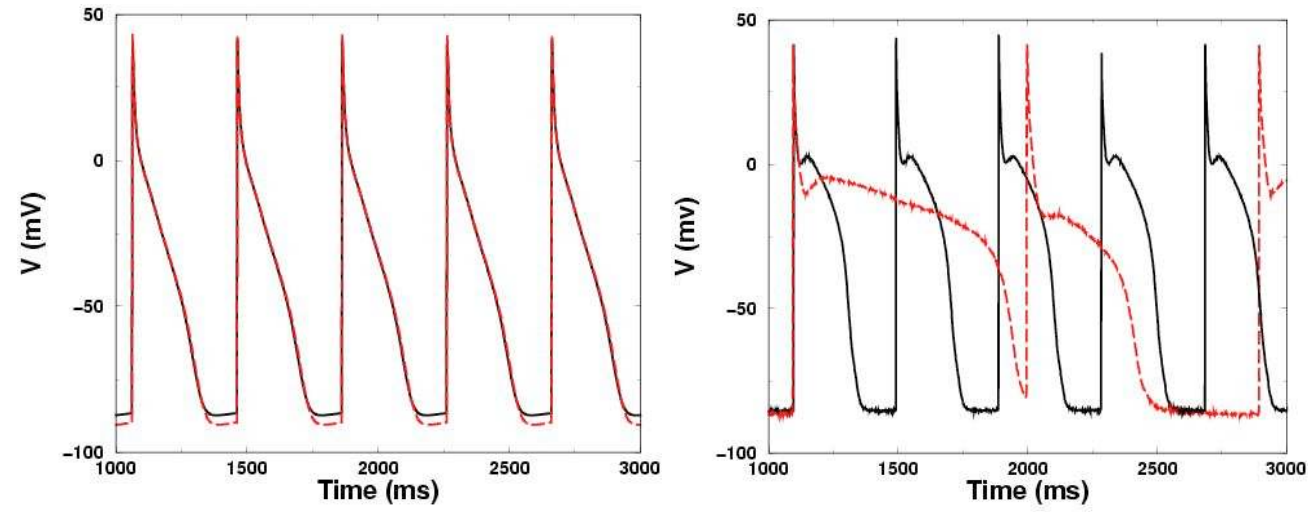

Figure 5. Left: Action potentials obtained using the DiFrancesco-Noble (DFN) Purkinje cell model paced at a cycle length of $400 \mathrm{~ms}$ with temperature set to $37^{\circ} \mathrm{C}$ (black) and $25^{\circ} \mathrm{C}$ (red). Right: Microelectrode recordings of canine Purkinje cells at a cycle length of $400 \mathrm{~ms}$ at a temperature of $37^{\circ} \mathrm{C}$ (black) and at a cycle length of $900 \mathrm{~ms}$ at a temperature of $25^{\circ} \mathrm{C}$ (red). At the lower temperature, action potentials prolong so dramatically that pacing at $400 \mathrm{~ms}$ no longer obtains a 1:1 response; in addition, pronounced alternans occurs at the relatively long cycle length of $900 \mathrm{~ms}$. 


\begin{tabular}{|c|c|c|c|c|c|c|}
\hline & CYTO & NSR & JSR & JS & SSS & TRPN \\
\hline Fox et al. 2002 & $\mathrm{X}$ & \multicolumn{2}{|c|}{$\mathrm{X}$} & & & \\
\hline Luo and Rudy 1994 & $\mathrm{X}$ & $\mathrm{X}$ & $\mathrm{X}$ & & & \\
\hline ten Tusscher and Panfilov 2006 & $\mathrm{X}$ & \multicolumn{2}{|c|}{$\mathrm{X}$} & $\mathrm{X}$ & & \\
\hline $\begin{array}{l}\text { Espinosa } 1998, \text { Hund and Rudy 2004*, Livshitz } \\
\text { and Rudy } 2007\end{array}$ & $\mathrm{X}$ & $\mathrm{X}$ & $\mathrm{X}$ & $\mathrm{X}$ & & \\
\hline Noble et al. 1991, Matsuoka et al. 2003 & $\mathrm{X}$ & $\mathrm{X}$ & $\mathrm{X}$ & & & $\mathrm{X}$ \\
\hline $\begin{array}{l}\text { Noble et al. 1998, Winslow et al. 1999, } \\
\text { Sakmann et al. 2000, Pandit et al. 2001, } \\
\text { Bondarenko et al. 2004, Iyer et al. } 2004\end{array}$ & $\mathrm{X}$ & $\mathrm{X}$ & $\mathrm{X}$ & $\mathrm{X}$ & & $\mathrm{X}$ \\
\hline Shannon et al. 2004 & $\mathrm{X}$ & \multicolumn{2}{|c|}{$\mathrm{X}$} & $\mathrm{X}$ & $\mathrm{X}$ & $\mathrm{X}^{* *}$ \\
\hline Mahajan et al. 2008 & $\mathrm{X}$ & $\mathrm{X}$ & $\mathrm{X}$ & $\mathrm{X}$ & $\mathrm{X}$ & $\mathrm{X}$ \\
\hline
\end{tabular}

* Volume of the junctional space actually coheres with the one that is generally referred to as subsarcolemmal space.

** A combined buffer that includes also troponin.

Table 1

A representative list of ventricular myocyte models classified according to the number of intracellular $\mathrm{Ca}^{2+}$ compartments. The six compartments refer to the bulk myoplasm (cytosol, CYTO); the network SR (NSR); the junctional SR (JSR), which refers to the releasable pool of $\mathrm{Ca}^{2+}$; junctional space (JS), the space between transmembrane $\mathrm{Ca}^{2+}$ channels and sarcoplasmic $\mathrm{Ca}^{2+}$ release channels; the sub-sarcolemmal space (SSS); and the $\mathrm{Ca}^{2+}$ bound to contractile elements (TRPN). 


\begin{tabular}{|c|c|c|c|c|c|}
\hline Protocol & $1 \mathrm{AP}$ & \multicolumn{3}{|c|}{ S1S2 Normal } & $\begin{array}{c}\text { S1S2 Norm. }+ \\
{[\mathrm{K}]_{\mathrm{o}}=4 \mathrm{mM}}\end{array}$ \\
\hline Data & Voltage & Voltage & Voltage $+[\mathrm{Ca}]_{\mathrm{i}}$ & $\begin{array}{c}\text { Voltage }+[\mathrm{Ca}]_{\mathrm{i}}+ \\
{[\mathrm{Ca}]_{\text {dyad }}}\end{array}$ & Voltage $+[\mathrm{Ca}]_{\mathrm{i}}$ \\
\hline \multirow[b]{2}{*}{$\begin{array}{l}\text { Identifiable } \\
\text { (of 12) }\end{array}$} & 4 & 8 & 10 & 12 & 12 \\
\hline & $\begin{array}{c}\mathrm{i}_{\mathrm{NaT}}, \\
\quad \mathrm{i}_{\mathrm{CaL}}, \mathrm{i}_{\mathrm{Ks}}, \\
\mathrm{i}_{\mathrm{Kto}}\end{array}$ & $\begin{array}{c}\mathrm{i}_{\mathrm{CaL}}, \mathrm{i}_{\mathrm{NaT}}, \mathrm{i}_{\mathrm{Ks}}, \\
\mathrm{NCX}, \mathrm{i}_{\mathrm{Ktoto}}, \\
\text { SERCA, } \mathrm{i}_{\mathrm{Kr}}, \\
\mathrm{i}_{\mathrm{NaK}}\end{array}$ & $\begin{array}{c}\mathrm{i}_{\mathrm{CaL}}, \mathrm{i}_{\mathrm{NaT}}, \mathrm{i}_{\mathrm{Ks}}, \\
\mathrm{SERCA}, \mathrm{NCX}, \\
\mathrm{i}_{\text {leak }}, \mathrm{i}_{\mathrm{Kto}}, \mathrm{i}_{\mathrm{NaK}}, \\
\mathrm{i}_{\mathrm{K} 1}, \mathrm{SR}^{\mathrm{xfer}}\end{array}$ & $\begin{array}{c}\mathrm{i}_{\mathrm{CaL}}, \mathrm{i}_{\mathrm{NaT}}, \mathrm{i}_{\mathrm{Ks}}, \\
\text { SERCA, } \mathrm{i}_{\mathrm{Kto}}, \\
\mathrm{SR}_{\mathrm{xfer}}, \mathrm{NCX}, \mathrm{i}_{\text {leak }}, \\
\mathrm{i}_{\mathrm{NaK}}, \mathrm{i}_{\mathrm{RvR}}, \mathrm{i}_{\mathrm{K} 1}, \mathrm{i}_{\mathrm{Kr}}\end{array}$ & $\begin{array}{c}\mathrm{i}_{\mathrm{NaT}}, \mathrm{i}_{\mathrm{CaL}}, \mathrm{i}_{\mathrm{K} 1}, \mathrm{i}_{\mathrm{Ks}}, \\
\text { SERCA, NCX, } \\
\mathrm{i}_{\mathrm{Kr}}, \mathrm{i}_{\text {leak }}, \mathrm{i}_{\mathrm{Kto}}, \\
\mathrm{SR}_{\mathrm{xfer}}, \mathrm{i}_{\mathrm{NaK}}, \mathrm{i}_{\mathrm{RvR}}\end{array}$ \\
\hline $\begin{array}{c}\text { Not } \\
\text { identifiable }\end{array}$ & $\begin{array}{c}\mathrm{i}_{\mathrm{Kr}}, \mathrm{i}_{\mathrm{K} 1}, \\
\text { SERCA, } \mathrm{i}_{\mathrm{NaK}}, \\
\mathrm{NCX}, \mathrm{SR}_{\mathrm{xfer}}, \\
\mathrm{i}_{\mathrm{RyR}}, \mathrm{i}_{\text {leak }}\end{array}$ & $\begin{array}{l}\mathrm{i}_{\mathrm{K} 1}, \mathrm{SR}_{\mathrm{xfer}}, \\
\mathrm{i}_{\text {leak }}, \mathrm{i}_{\mathrm{RyR}}\end{array}$ & $\mathrm{i}_{\mathrm{Kr}}, \mathrm{i}_{\mathrm{RyR}}$ & & \\
\hline
\end{tabular}

$\mathrm{i}_{\mathrm{NaT}}$ fast transient $\mathrm{Na}^{+}$current; $\mathrm{i}_{\mathrm{CaL}} \mathrm{L}$-type $\mathrm{Ca}^{2+}$ current; $\mathrm{i}_{\mathrm{K} 1}$ inwardly rectifying $\mathrm{K}^{+}$current; $\mathrm{i}_{\mathrm{Kr}}$ rapidly inactivating inwardly rectifying $\mathrm{K}^{+}$current; $\mathrm{i}_{\mathrm{Ks}}$ rapidly inactivating inwardly rectifying $\mathrm{K}^{+}$current; $\mathrm{i}_{\mathrm{Kto}}$ transient outward $\mathrm{K}^{+}$current; SERCA sarcoplascmic reticulum (SR) $\mathrm{Ca}^{2+}$ ATPase; $\mathrm{NCX} \mathrm{Na} / \mathrm{Ca}^{2+}$ exchanger; $i_{\mathrm{NaK}} \mathrm{Na}^{+} / \mathrm{K}^{+}$pump; $i_{\mathrm{RyR}}$ ryanodine receptor SR release current; $i_{\text {leak }}$ SR leak current; $\mathrm{SR}_{\mathrm{xfer}}$ diffusive current from network SR to junctional SR.

Table 2: Identifiability of ion channel conductances and maximal rates for pumps and exchangers from measurements using an S1S2-protocol. Note that the ordering of the identifiable parameters is model dependent. 


\begin{tabular}{|l|c|c|c|c|c|}
\hline Cell Model & Rabbit & Human & Mouse & Canine & Guinea Pig \\
\hline \hline 1Hz APD $90(\mathrm{~ms})$ & 215 & 304 & 19 & 235 & 159 \\
\hline \multicolumn{5}{|c|}{ \% Change } \\
\hline \\
\hline \multirow{2}{|l|}{ Pacing Rate } & & & & & \\
\hline $0.5 \mathrm{~Hz}$ & 3.3 & 2.3 & 0.0 & 4.3 & 8.8 \\
$2.0 \mathrm{~Hz}$ & -12.1 & -8.9 & -5.3 & -5.5 & -15.2 \\
\hline Extracellular $\left[\mathrm{Ca}^{2+}\right]_{0}$ & & & & & \\
\hline \hline $2.5 \mathrm{mM}$ & 19.5 & 5.3 & 0.0 & -6.4 & 3.8 \\
$3.0 \mathrm{mM}$ & 40.0 & 10.2 & -5.3 & - & 6.3 \\
\hline Extracellular $\left[\mathrm{K}^{+}\right]_{0}$ & & & & & \\
\hline \hline $8 \mathrm{mM}$ & 0.0 & -0.7 & 10.5 & -14.0 & 3.8 \\
$10 \mathrm{mM}$ & -3.7 & -2.0 & - & -18.7 & 3.1 \\
\hline
\end{tabular}

Table 3: Rabbit (Shannon et al. 2004), human (ten Tusscher et al. 2004), mouse (Bondarenko et al. 2004), canine (Hund and Rudy 2004) and guinea pig (Livshitz and Rudy 2007) cell cardiac cell models simulated under different conditions. All models solved to limit cycle in Cellular Open Resource (Garny et al. 2003). In cases where the stimulus current was not accounted for in the original model it was added as a $\mathrm{K}^{+}$current. Dashes indicate cases where the model did not reach a limit cycle. 